\title{
Pathophysiologische Veränderungen spezifischer neurofunktioneller Systeme bei Schizophrenie
}

\author{
INAUG URAL-DISSERTATION \\ zur Erlangung des D oktorgrades \\ der Medizinischen Fakultät der \\ G eorg-August-Universität zu G öttingen
}

vorgelegt von

Aleksandra D echent

aus

Frankfurt am Main

Göttingen 2019 
D ekan:

Referent:

Ko-Referent/ in:

D rittreferent/ in:
Prof. D r. med. W. Brück

Prof. D r. med. O. G ruber

Prof. D r. rer. nat. M. Wilke 
Hiermit erkläre ich, die Dissertation mit dem Titel "Pathophysiologische Veränderungen spezifischer neurofunktioneller Systeme bei Schizophrenie" eigenständig angefertigt und keine anderen als die von mir angegebenen Quellen und Hilfsmittel verwendet zu haben.

Göttingen, den ..............

(Unterschrift) 


\section{Inhaltsverzeichnis}

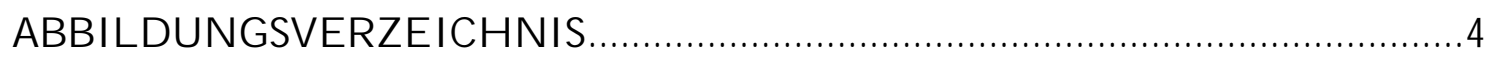

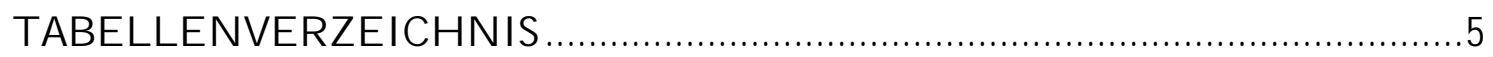



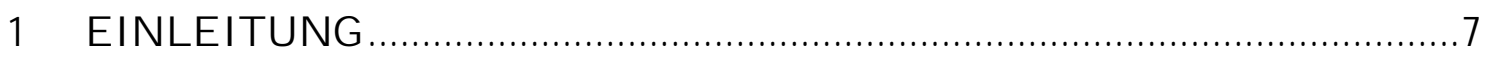

1.1 Allgemeiner wissenschaftlicher Hintergnund ...................................................7

1.2 Grundlagenwissen zur Schizophrenie .................................................................8

1.2.1 Epidemiologie .................................................................................................

1.2.2 Diagnostik und klinisches Erscheinungsbild .....................................................

1.2.3 Ätiologie und Pathophysiologie ..........................................................................10

1.2.4 Subtypen der Schizophrenie ..............................................................................

1.2.5 Verlauf und Prognose..............................................................................................12

1.2.6 Therapie .......................................................................................................12

1.2.7 Schizoaffektive Störung.........................................................................................13

1.3 Das dopaminerge System und pathophysiologische Veränderungen bei

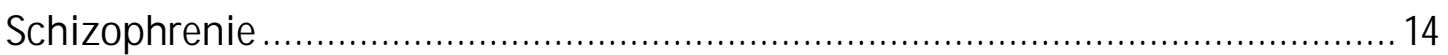



2 MATERIAL UND METHODEN ........................................................................ 18

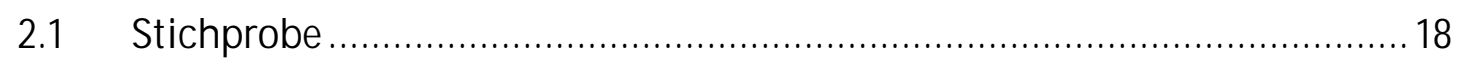

2.2 Psychopathologische Skalen ......................................................................20

2.2.1 Positive and N egative Syndrome Scale (PANSS) ......................................................20 
2.2.2 Clinical G lobal Impression Scale (CG I)....................................................................20

2.2.3 M ontgomery-A sberg D epression Scale (MAD RS) ........................................................21

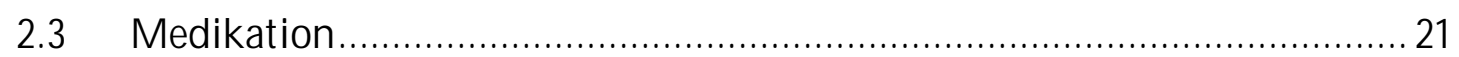

2.4 Magnetresonanztomografie (MRT) ............................................................22

2.4.1 Funktionelle Magnetresonanztomografie (fMRT)...........................................23

2.5 Paradigma

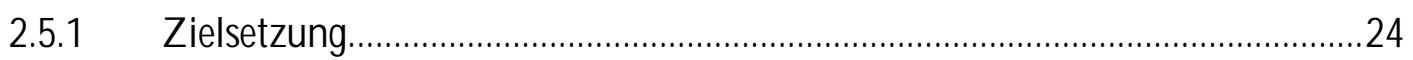

2.5.2 D urchführung ................................................................................................24

2.5.2.1 O perante Konditionierung............................................................................2

2.5.2.2 Experimentelle Verhaltensaufgabe .............................................................25

2.5.3 fMRT-Untersuchung...........................................................................................2

$2.6 \quad$ Datenanalyse................................................................................................3

2.6.1 Analyse der Performanzdaten..............................................................................30

2.6.2 Analyse der fMRT-D aten ...............................................................................30

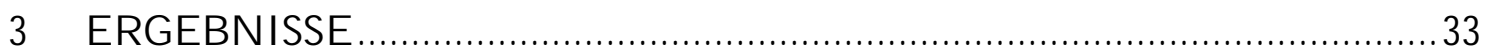

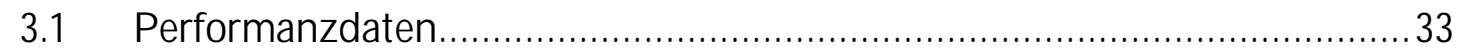

3.1.1 D esire Kontext ...............................................................................................

3.1.2 Reason-Kontext...............................................................................................



3.2.1 D esire-Kontext......................................................................................................

3.2.2 Reason-Kontext..................................................................................................40

3.2.3 Psychophysiologische Interaktion (PPI) ............................................................4

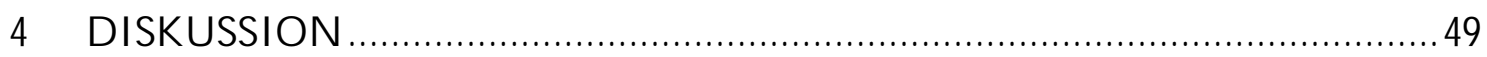

4.1 Mesolimbisches System - bottom-up-Regulation ............................................49 
$4.2 \quad$ Frontalkortex - top-down-Regulation...............................................52

4.3 Methodische Aspekte ............................................................................5



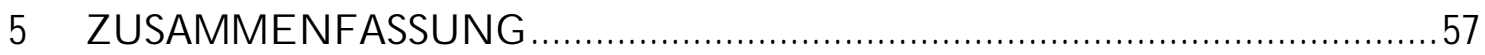



6.1 Messparameter für die Magnetresonanztomografie .................................59

6.2 Fragebögen ....................................................................................................62 62

6.2.1 Positive and N egative Syndrome Scale (PANSS) .........................................................62

6.2.2 Clinical G lobal Impression Scale (CG I)...........................................................63

6.2.3 M ontgomery-A sberg D epression Scale (MAD RS) .....................................................65

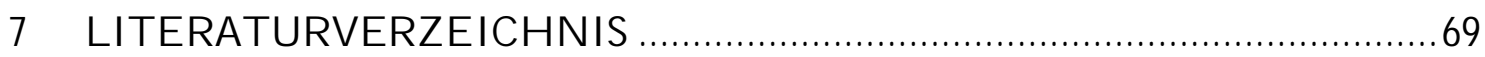




\section{Abbildungsverzeichnis}

Abbildung 1: D as dopaminerge System. ………………………………………………….....15

Abbildung 2: Paradigma: Schematischer Ablauf eines Aufgabenblocks „Zielverfolgung“.

Abbildung 3: Paradigma: Schematischer Ablauf eines Aufgabenblocks „Bonusverfolgung“.

Abbildung 4: G esteigerte Aktivierung im Aufgabenblock „Bonusverfolgung“ bei Patienten

im Vergleich zu Kontrollpersonen. 39

Abbildung 5: G esteigerte Aktivierung im Aufgabenblock „Zielverfolgung“ bei Patienten im Vergleich zu Kontrollpersonen. . .45

Abbildung 6: Die PPI-Analyse zeigt eine reduzierte negative funktionelle Interaktion bei Patienten im Vergleich zu Kontrollen in der Dilemmasituation. 


\section{Tabellenverzeichnis}

Tabelle 1: Kriterien zur Diagnosesicherung der Schizophrenie entsprechend ICD-10..........9

Tabelle 2: Subtypen der Schizophrenie entsprechend ICD -10...............................................11

Tabelle 3: Kriterien zur Diagnosesicherung der schizoaffektiven Störung entsprechend ICD -10 .

Tabelle 4: Ein- und Ausschlusskriterien für Patienten..........................................................18

Tabelle 5: Soziodemographische Daten der Stichprobe......................................................19

Tabelle 6: Psychopathologische Skalen der Patienten.............................................................21

Tabelle 7: Medikation der Patienten. .....................................................................................22

Tabelle 8: Verhaltensdaten der Stichprobe..............................................................................34

Tabelle 9: Aktivierungen der Patienten und Kontrollen im Aufgabenblock

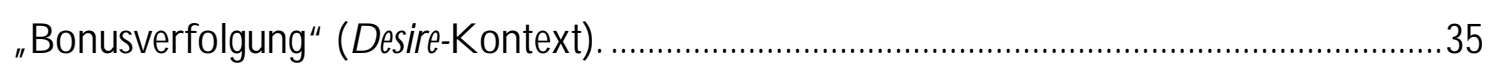

Tabelle 10: Differentielle Aktivierungen zwischen Patienten und Kontrollen im A ufgabenblock „Bonusverfolgung" (D esire-Kontext)...........................................................38

Tabelle 11: Aktivierungen der Patienten und Kontrollen im Aufgabenblock

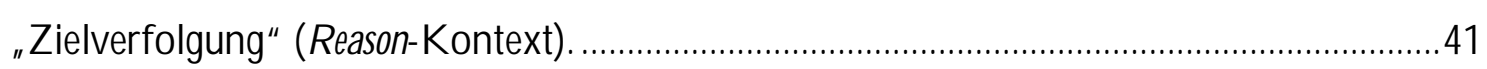

Tabelle 12: Differentielle A ktivierungen zwischen Patienten und Kontrollen im Aufgabenblock „Zielverfolgung“ (Reason-Kontext)................................................................4

Tabelle 13: G esteigerte negative funktionelle Interaktion bei Kontrollen in der Dilemmasituation, ausgehend von Regionen im ventralen Striatum........................................46

Tabelle 14: Reduzierte negative funktionelle Interaktion bei Patienten im Vergleich zu Kontrollen in der D ilemmasituation, ausgehend von Regionen im ventralen Striatum.......47 


\section{Abkürzungsverzeichnis}

\begin{tabular}{|c|c|}
\hline ANOVA & A nalysis of $\mathrm{V}$ ariance \\
\hline avPFC & antero-ventraler präfrontaler Kortex \\
\hline BOLD & Blood $0 x$ ygenation L evel D ependent \\
\hline CG I & Clinical G lobal Impression Scale \\
\hline D RD -Paradigma & D esire-Reason-D ilemma-Paradigma \\
\hline GLM & G eneral L inear M odel (Allgemeines lineares Modell) \\
\hline ICD -10 & $\begin{array}{l}\text { International Statistical Classification of D iseases and R elated H ealth Problems } \\
\text { (Version 2016) }\end{array}$ \\
\hline fMRT & funktionelle Magnetresonanztomografie \\
\hline MRT & Magnetresonanztomografie \\
\hline MADRS & M ontgomery-A sberg D epression Scale \\
\hline MW & Mittelwert \\
\hline $\mathrm{Nacc}$ & Nucleus accumbens \\
\hline PANSS & Positive and N egative Syndrome Scale \\
\hline PPI & psychophysiologische Interaktionsanalyse \\
\hline SD & Standardabweichung \\
\hline SVC & Small V olume Correction (Korrektur für kleine Volumina) \\
\hline VMPFC & ventraler medialer präfrontaler Kortex \\
\hline vStr & ventrales Striatum \\
\hline VTA & ventrale tegmentale Area \\
\hline
\end{tabular}




\section{Einleitung}

\subsection{Allgemeiner wissenschaftlicher Hintergnund}

Die Schizophrenie ist ein häufiges psychiatrisches Erkrankungsbild, mit stark heterogener Klinik und Ausprägung, dessen Ätiologie noch weitgehend unbekannt ist. Bereits 1911 beschrieb Eugen Bleuler ein sog. Spaltungsirresein (Bleuler 1911; Moskowitz und Heim 2011), welches die Bezeichnung Schizophrenie erklärt (griechisch: Schizo - ich spalte, phren Geist). Hierunter versteht man eine Spaltung des Denkens, Fühlens und des eigenen subjektiven Empfindens der Persönlichkeit.

Nach derzeitiger Klassifikation werden verschiedene Krankheitsbilder entsprechend ICD10 eingeteilt. Patienten werden anhand von klinischen Symptomen in die D iagnosegruppe der Schizophrenie eingeteilt. Aufgrund der komplexen Manifestation der Schizophrenie gestaltet sich eine Diagnosestellung oftmals sehr schwierig, da pathognomische Symptome oft fehlen (Pearlson und Calhoun 2009). Auch innerhalb einer als schizophren diagnostizierten $\mathrm{G}$ ruppe scheint es Untergruppen zu geben. Eine Therapie gestaltet sich oft langwierig und erschwert.

Durch Untersuchungen mit bildgebenden Verfahren, unter anderem mit funktioneller Magnetresonanztomografie (fMRT), konnten Funktionen und Dysfunktionen neuronaler Systeme identifiziert werden (G ruber 2001; Gruber und von Cramon 2003; Melcher und Gruber 2006). Hierbei wurde in jüngster Vergangenheit zunehmend auf neuronale Netzwerke geachtet, welche in grundlegende kognitive Funktionen involviert sind und bei Schizophrenen Dysfunktionen zeigen. Ein neuronales System, welches im Zusammenhang mit der Schizophrenie von besonderer Bedeutung $\mathrm{zu}$ sein scheint, ist das Belohnungssystem bzw. das mesolimbische System.

Weiterführende Untersuchungen zur Identifizierung von für die Schizophrenie typischen Dysfunktionen in neuronalen Netzwerken sind von erheblicher Wichtigkeit, um in Zukunft die Diagnosestellung Schizophrenie zu erleichtern und somit ein frühes Erkennen und Behandeln der Patienten zu ermöglichen. Dadurch würde nicht nur das subjektive Leidensempfinden der Patienten reduziert, sondern auch das Gesundheitssystem entlastet.

Entsprechend untersucht die vorliegende Studie spezifische neurofunktionelle Systeme, welche maßgebliche Dysfunktionen bei Patienten mit Schizophrenie zeigen, mittels fMRT und speziellen Verhaltensparadigmen. 


\subsection{Grundlagenwissen zur Schizophrenie}

\subsubsection{E pidemiologie}

Die Wahrscheinlichkeit, im Leben einmal an einer schizophrenen Episode zu erkranken, beträgt 0,5 bis 1,6\% und somit zählt die Schizophrenie zu den häufigsten Psychoseformen (Shenton et al. 2001). Die Prävalenz beträgt weltweit 0,1 bis $1 \%$. Die Geschlechterverteilung ist ausgeglichen, wobei Männer im Schnitt fünf Jahre früher erkranken. Die Erkrankung manifestiert sich vor allem im frühen bis mittleren Lebensabschnitt, also zwischen dem 20. und 30. Lebensjahr. Ein zweiter Häufigkeitsgipfel wird bei Frauen während der (Prä)Menopause beschrieben, also zwischen dem 45. und 49. Lebensjahr (Maurer und Häfner 2002).

\subsubsection{Diagnostik und klinisches E rscheinungsbild}

Wie bereits erwähnt, handelt es sich bei der Schizophrenie um eine sehr schwere psychiatrische Erkrankung, die durch eine extrem heterogene Symptomatik gekennzeichnet ist (Kendler et al. 1998; Möller et al. 2011). Bei Schizophrenen zeigen sich eine Vielzahl an psychischen Störungen, wie die Beeinträchtigung von Aufmerksamkeit, Konzentration, Antrieb, Affekt, formalem und inhaltlichem Denken, Ich-Erleben sowie von Psychomotorik. Nach K.H. Jackson werden die Symptome in Positiv- und Negativsymptome eingeteilt, deren Ausmaß anhand der PANSS (Positive and Negative Syndrome Scale) erfasst werden kann. Darüberhinaus können weitere psychopathologische Skalen eingesetzt werden, um z. B. den allgemeinen Schweregrad der Erkrankung unabhängig von der Diagnose (Clinical G lobal Impression Scale (CGI)) oder die Schwere der depressiven Symptome des Patienten (M ontgomery-A sberg Depression Scale (MADRS)) zu erfassen.

$\mathrm{Zu}$ den Positivsymptomen zählen unter anderem Halluzinationen, Wahn und formale D enkstörungen. Negativsymptome beinhalten Störungen des Antriebs, Affektverflachung, Störung der Affektivität und Psychomotorik sowie Antriebsarmut. Die Intelligenz ist in aller Regel nicht betroffen, zumindest zu Beginn der Manifestation (Möller 2015).

Die Kriterien zur Diagnosesicherung der Schizophrenie entsprechend ICD-10 sind in Tabelle 1 dargestellt. 
Tabelle 1: Kriterien zur Diagnosesicherung der Schizophrenie entsprechend ICD-10 (aus Marneros (2011)).

\section{Eingangskniterien}

Während eines Zeitraums von mindestens einem Monat sollte eine psychotische Episode bestehen mit:

\begin{tabular}{|c|c|}
\hline $\begin{array}{l}\text { mindestens einem der } \\
\text { folgenden Merkmale }\end{array}$ & $\begin{array}{l}\text { - Gedankenlautwerden, -eingebung, -entzug oder -ausbreitung } \\
\text { - Kontroll-, Beeinflussungswahn, Gefühl des Gemachten, } \\
\text { Wahnwahmehmung } \\
\text { - Kommentierende oder dialogische Stimmen, oder andere } \\
\text { Stimmen, die aus bestimmten Körperteilen kommen } \\
\text { - Anhaltender kulturell unangemessener Wahn }\end{array}$ \\
\hline $\begin{array}{l}\text { ODER } \\
\text { mindestens zweien } \\
\text { der folgenden Merk- } \\
\text { male }\end{array}$ & $\begin{array}{l}\text { - Anhaltende Halluzinationen jeder Sinnesmodalität, begleitet von } \\
\text { flüchtigen Wahngedanken oder von langanhaltenden } \\
\text { überwertigen Ideen } \\
\text { - Gendankenabreißen oder Einschiebungen in den } \\
\text { Gedankenfluss, was zu Zerfahrenheit, Neologismen oder } \\
\text { Danebenreden führt } \\
\text { - Katatone Symptome (wie Erregung, Haltungsstereotypien, } \\
\text { wächserne Biegsamkeit, Negativismus, Mutismus, Stupor) } \\
\text { - „negative“ Symptome (wie Apathie, Sprachverarmung, } \\
\text { verflachte oder inadäquate Affekte) }\end{array}$ \\
\hline
\end{tabular}

\section{Häufigste Ausschlusskriterien}

- Die Störung kann einer organischen Hirnerkrankung (F00-F09) oder einer Störung durch psychotrope Substanzen (F1) zugeordnet werden. 


\subsection{3 Ätiologie und Pathophysiologie}

Es liegt nahe, dass es sich bei der Schizophrenie um eine multifaktorielle, heterogene Erkrankung handelt (Möller et al. 2011). Die genaue Entstehung ist größtenteils noch unbekannt, wobei eine genetische Prädisposition jedoch sicherlich von Bedeutung ist. So ließ sich in der Vergangenheit nachweisen, dass das Erkrankungsrisiko für Menschen, die mit einer an Schizophrenie leidenden Person verwandt sind, umso größer ist, je höher der Grad der Verwandschaft ist (Kendler et al. 1998). Auch konnte gezeigt werden, dass bei eineiigen Zwillingen die Konkordanzrate bei 50\% liegt, bei zweieiigen Zwillingen liegt sie bei 10\% (Tandon et al. 2008). D a jedoch nur ca. 20\% der Schizophrenien familiär gehäuft vorkommen, müssen auch andere Einflussfaktoren mit berücksichtigt werden. Entsprechend muss also von einer multifaktoriellen Äthiopathogenese ausgegangen werden.

Ein besonderes Augenmerk richtete sich auf das Vulnerabilitäts-Stress-Coping-Modell. In diesem werden sowohl genetisch-biologische als auch psychosoziale Faktoren mit einbezogen. Bei genetischer Prädisposition können bestimmte exogene Einflüsse, wie zum Beispiel Geburtskomplikationen, das Entstehen einer Schizophrenie begünstigen (Rapoport et al. 2005). Auch weitere Faktoren wie Drogen- oder Alkoholabusus sowie Stress können die Erkrankung begünstigen. Es ist jedoch möglich, durch Coping Strategien oder positive exogene Faktoren den Verlauf der Erkrankung zu verbessern (Möller et al. 2011).

\subsubsection{Subtypen der Schizophrenie}

Aufgrund der ausgeprägten Inhomogenität des Erscheinungsbildes der Schizophrenie versucht man, anhand der dominierenden Symptomatik Patienten in Subgruppen einzuteilen. Um einen Überblick zu geben, ist in Tabelle 2 die klassische Subtypisierung dargestellt. 
Tabelle 2: Subtypen der Schizophrenie entsprechend ICD -10 (aus Möller et al. (2011)).

F20: Schizophrenie

\begin{tabular}{|c|c|}
\hline $\begin{array}{l}\text { F20.0 } \\
\text { Paranoide } \\
\text { Schizophrenie }\end{array}$ & $\begin{array}{l}\text { Positivsymptomatik wie Halluzinationen und Wahn stehen im } \\
\text { Vordergrund. Diese Form ist der häufigste Typ. }\end{array}$ \\
\hline $\begin{array}{l}\text { F20.1 } \\
\text { Hebephrene } \\
\text { Schizophrenie }\end{array}$ & $\begin{array}{l}\text { Leitende Symptome sind vor allem formale D enkstörungen, Störungen } \\
\text { des Antriebs und Affekts, oftmals auch enthemmtes Sozialverhalten. } \\
\text { Dieser Typ findet sich gehäuft bei Jugendlichen. }\end{array}$ \\
\hline $\begin{array}{l}\text { F20.2 } \\
\text { Katatone } \\
\text { Schizophrenie }\end{array}$ & $\begin{array}{l}\text { Bei diesem Subtypen kann eine Störung der Psychomotorik von } \\
\text { Stupor bis Hyperkinesie reichen. Es handelt sich um einen eher } \\
\text { seltenen Subtyp. }\end{array}$ \\
\hline $\begin{array}{l}\text { F20.3 } \\
\text { Undifferenzierte } \\
\text { Schizophrenie }\end{array}$ & $\begin{array}{l}\text { Diese Subtypisierung bezieht sich nur auf Erkrankte die in keine } \\
\text { andere Subgnuppe passen und beschreibt immer eine akute } \\
\text { Schizophrenie. }\end{array}$ \\
\hline $\begin{array}{l}\text { F20.4 } \\
\text { Postschizophrene } \\
\text { Depression }\end{array}$ & $\begin{array}{l}\text { Diese Gruppe schließt Patienten ein, welche nach einem akuten } \\
\text { schizophrenem Schub eine Depression entwickeln. Während der } \\
\text { depressiven Phase herrschen weiter schizophrene Symptome vor, im } \\
\text { Vordergrund steht jedoch die depressive Symptomatik. }\end{array}$ \\
\hline $\begin{array}{l}\text { F20.5 } \\
\text { Schizophrenes } \\
\text { Residuum }\end{array}$ & $\begin{array}{l}\text { Nach stattgehabter schizophrener Episode verbleibt eine } \\
\text { Negativsymptomatik. }\end{array}$ \\
\hline $\begin{array}{l}\text { F20.6 } \\
\text { Schizophrenia } \\
\text { simplex }\end{array}$ & $\begin{array}{l}\text { Hier zeigt sich eine zunehmende Negativsymptomatik ohne vorherige } \\
\text { psychotische Symptome. }\end{array}$ \\
\hline $\begin{array}{l}\text { F20.8 } \\
\text { Sonstige } \\
\text { Schizophrenie }\end{array}$ & \\
\hline
\end{tabular}




\subsubsection{Verlauf und Prognose}

D ie Manifestation einer Schizophrenie verläuft typischerweise in mehreren Phasen (Möller 2015). Initial unterscheidet man eine prämorbide Phase, welche sich bereits in der Kindheit bemerkbar macht und mit kognitiven, motorischen oder sozialen Defiziten einhergeht. D ieser folgt eine Prodromalphase, welche durch Negativsymptomatik sowie abgeschwächte psychotische Symptome gekennzeichnet ist. Jedoch entwickeln weniger als $50 \%$ dieser Patienten im Verlauf eine manifeste Schizophrenie. An diese Phase schließt sich die psychotische Phase an, welche mit einer ausgeprägten Positivsymptomatik einhergeht und den klassischen Beginn einer Schizophrenie charakterisiert. Nach etwa zehn Jahren wird eine stabile Phase erreicht, in der Negativsymptome das Ausmaß der Erkrankung dominieren. Ungefähr ein Viertel aller Patienten erreicht komplette psychopathologische Remission und 50\% aller Patienten eine soziale Remission. Eine frühzeitige antipsychotische Behandlung zeigt eine bessere Prognose (Tandon et al. 2009). Um etwa 15 bis 20 Jahre verkürzt sich die durchschnittliche Lebenserwartung bei an Schizophrenie erkrankten Personen, unter anderem bedingt durch eine erhöhte Suizidrate (5\%) sowie durch weitere, z. B. kardiovaskuläre Risikofaktoren und indirekte Faktoren wie Nikotinabusus, C2-A busus und ähnliches (Tandon et al. 2009). Neben dieser typischen Manifestation gibt es weitere Subtypen der Schizophrenie, welche sich in Verlauf und Prognose unterscheiden können.

\subsubsection{Therapie}

In der Vergangenheit hat sich ein mehrdimensionales Konzept zur Behandlung der Schizophrenie durchgesetzt (Möller et al. 2011). Eine wichtige Rolle spielt auch die Psychotherapie, insbesondere die Durchführung von kognitiv-behavioralen Maßnahmen, sowie die Psychoedukation und familentherapeutische Maßnahmen. D es Weiteren wird die pharmakologische Behandlung mit Neuroleptika eingesetzt. Neben den klassischen Neuroleptika wie Haloperidol, gewinnen vor allem die neueren, atypischen Neuroleptika wie Clozapin, Risperidon, Olanzapin zunehmend an Bedeutung. Dies ist maßgeblich auf die deutlich weniger bis gar nicht vorkommenden extrapyramidalen Nebenwirkungen zurückzuführen. Die Auswahl eines passenden Neuroleptikums bei Erstmanifestation der Erkrankung gestaltet sich - trotz Verfügbarkeit zahlreicher Studien - als schwierig (Tandon et al. 2010). D a der Erfolg eines bestimmten Neuroleptikums nicht sicher ist, erfolgt die endgültige Auswahl nach wie vor nach dem Trial-and-E rror-Prinzip (Gruber und Falkai 2009). 


\subsubsection{Schizoaffektive Stönung}

Da in der hier vorliegenden Studie auch einige wenige Patienten mit der Diagnose einer schizoaffektiven Störung eingeschlossen wurden, soll im Folgenden kurz auf dieses Krankheitsbild eingegangen werden. Die schizoaffektive Störung beeinhaltet sowohl Symptomeigenschaften des Erkrankungsbildes der Schizophrenie als auch der monopolaren oder bipolaren affektiven Störung. D abei unterscheidet man drei Formen der schizoaffektiven Störung: die schizoaffektive Störung mit manischer Ausprägung (schizomanische Form), die schizoaffektive Störung mit depressiver Ausprägung (schizodepressive Form) sowie eine gemischte schizoaffektive Störung. Der Krankheitsverlauf ist bei den monopolaren Formen meist oligophasisch mit weniger als drei Episoden, während die bipolare Form meist einen polyphasischVerlauf mit mehr als drei Episoden aufweist. Die Häufigkeit persistierender Alterationen nach einer schizoaffektiven Störung ist höher als nach einer affektiven Erkrankung, aber niedriger als nach einer schizophrenen Erkrankung. Um nach ICD-Kriterien die Diagnose einer schizoaffektiven Störung stellen zu können, muss neben einer affektiven Störung, ein weiteres der unten aufgeführten Kriterien während der Störungspahse erfüllt sein (Tabelle 3). Die Behandlung der schizoaffektiven Erkrankung richtet sich nach der Form und der jeweiligen Schwere des Erscheinungsbildes. Schizomanische Formen werden mit Antipsychotika behandelt, während bei der schizodepressiven Form eine Kombination aus Antipsychotika und Antidepressiva zum Einsatz kommt (Marneros 2011). 
Tabelle 3: Kriterien zur Diagnosesicherung der schizoaffektiven Störung entsprechend ICD -10 (aus Marneros (2011)).

\section{Eingangskniterien}

Die Kriterien für eine affektive Störung (F30-F32) mit mittelgradigem oder schwerem Schweregrad sind erfüllt.

Über einen Zeitraum von mindestens 2 Wochen müssen während des größten Teils dieser Zeit Symptome aus mindestens einer der folgenden Symptomgruppen (entsprechen nahezu denen der Schizophrenie (F20.0-20.3); vgl. Tabelle 1) vorhanden sein:

- Gedankenlautwerden, -eingebung, -entzug oder -ausbreitung

- Kontroll-, Beeinflussungswahn, G efühl des Gemachten, Wahnwahmehmung

- Kommentierende oder dialogische Stimmen, oder andere Stimmen, die aus bestimmten Körperteilen kommen

- Anhaltender kulturell unangemessener Wahn

- Gendankenabreißen oder Einschiebungen in den Gedankenfluss, was zu Zerfahrenheit, Neologismen oder Danebenreden führt

- Katatone Symptome (wie Erregung, Haltungsstereotypien, wächseme Biegsamkeit, Negativismus, Mutismus, Stupor)

Diese Kriterien müssen während derselben Störungsepisode und zeitweise gleichzeitig erfüllt sein.

\section{Häufigste Ausschlusskriterien}

- Die Störung kann einer organischen Hirnerkrankung (F00-F09) oder einer Störung durch psychotrope Substanzen (F1) zugeordnet werden.

\subsection{Das dopaminenge System und pathophysiologische Ver- änderungen bei Schizophrenie}

Um seinen eigenen Fortbestand zu sichem, muss der Mensch immer wieder neue Entscheidungen treffen, welche zum Teil weitreichende Folgen nach sich ziehen. Es gilt dabei abzuwägen, ob eine Entscheidung einen Vorteil oder einen Nachteil birgt. Belohnungen dienen hierbei als Anreiz und sind von großer Bedeutung für zielgerichtetes 
Verhalten (Haber und Knutson 2010). Ein neuroanatomisches Korrelat hierfür ist das dopaminerge Belohnungssystem (Abbildung 1), auf welches im Folgenden näher eingegangen werden soll.

Als zentrales Element des Belohnungssystems ist der Nucleus accumbens (Nacc) anzusehen, welcher durch dopaminerge Fasem der ventralen tegmentalen Area (VTA) innerviert wird (O'Connell und Hofmann 2011). Weiter zählen zum Belohnungssystem der präfrontrale Kortex, der orbitofrontale Kortex, das ventrale Striatum (vStr) sowie der anteriore cinguläre Kortex. In hirnbildgebenden Studien mit Probanden, welchen belohnende Stimuli angeboten wurden, zeigte sich in diesen Arealen des Belohnungssystems eine Aktivierung (Knutson et al. 2001). Weitere Areale, welche ebenfalls eine Aktivierung zeigten und mutmaßlich auch als regulierende Strukturen anzusehen sind, sind der Hippocampus, die bilaterale Amygdala, der laterale habenuläre Nucleus sowie die Raphekerne.

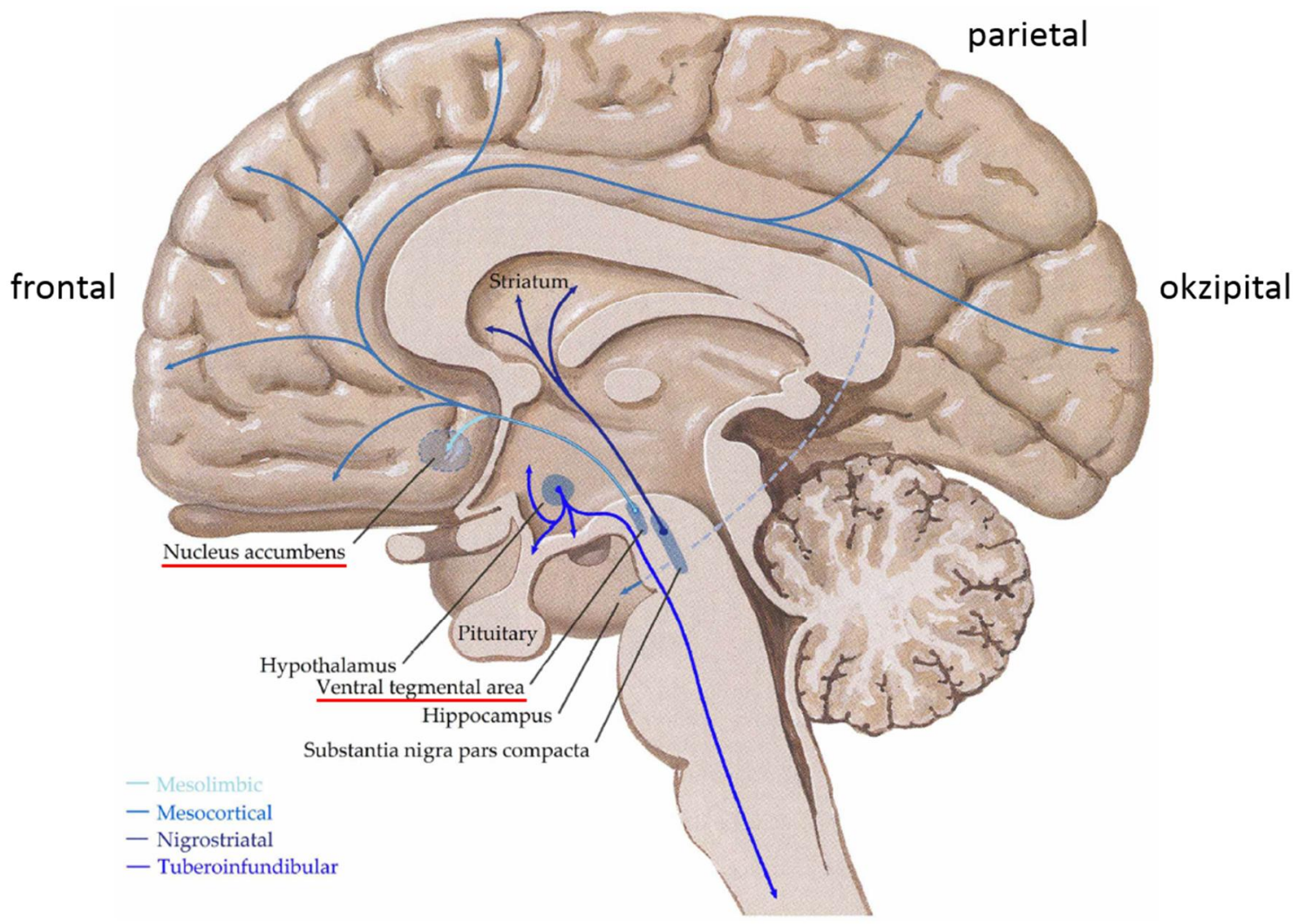

Abbildung 1: Das dopaminerge System. Nähere Erläuterungen siehe Text (verändert aus Scarr et al. (2013)). 
Um das Überleben zu sichem, muss der Mensch gelegentlich unmittelbare Belohnungen zu G unsten von längerfristigen Zielen zurückstellen. Es liegt nahe, dass übergeordnete Schaltsysteme existieren, welche das Bedürfnis, eine Belohnung zu erhalten, mit modulieren bzw. einschränken. In einer 2010 durchgeführten Studie gelang es Diekhof und G ruber, dies neurowissenschaftlich zu bestätigen (D iekhof und Gruber 2010). Probanden wurden in einer fMRT-Studie belohnende Stimuli dargeboten, welche entweder direkt angenommen oder aber zu Gunsten eines übergeordneten Ziels abgelehnt werden sollten (Dilemmasituation). Hierbei zeigte sich, wenn die Probanden versuchten, übergeordnete Ziele zu erreichen, eine negative Interaktion des antero-ventralen präfrontalen Kortex (avPFC) mit dem Nacc sowie der VTA (D iekhof und Gruber 2010). Daraus schlossen die Autoren, dass der avPFC einen modulierenden Einfluss auf den Nacc und die VTA besitzt. Seit Längerem wird gemutmaßt, dass in diesen funktionellen Systemen bei an Schizophrenie erkrankten Personen ein Ungleichgewicht vorliegt. Es wird angenommen, dass sowohl eine frontale dopaminerge Hypoaktivität als auch eine mesolimbische dopaminerge Hyperaktivität besteht. Während ersteres für die Negativsymptomatik verantwortlich sein soll, soll letzteres für die Positivsymptomatik ausschlaggebend sein (Möller et al. 2011).

Es ist daher naheliegend, dass, wenn es um die grundsätzliche Ursachenfindung und die Identifikation von neuropathophysiologischen Netzwerken bei an Schizophrenie erkrankten Personen geht, diese Strukturen und ihr Ungleichgewicht von zentraler Bedeutung sind.

\subsection{Zielsetzung}

Das primäre Ziel dieser Arbeit ist es, die Dysfunktion des dopaminergen Belohnungssystems bei an Schizophrenie erkrankten Patienten durch Einsatz der fMRT weiter zu charakterisieren. Hierzu ist es zunächst notwendig die neuronalen Netzwerke zu identifizieren, welche bereits durch vorherige Studien als zentrale Strukturen des dopaminergen Belohnungssystems belegt wurden. D urch die Kombination von fMRT und einem speziellen Verhaltensparadigma, dem Desire Reason-D ilemma (D RD)-Paradigma (Diekhof und Gruber 2010), sollen zunächst spezifische Aktivierungen in denjenigen A realen identifiziert werden, welche an Entscheidungsprozessen und Belohnungsverarbeitung mitbeteiligt sind. Im Rahmen des eingesetzten D RD-Paradigmas 
wird eine Dilemmasituation herbeigeführt. Während einer Teilaufgabe des Paradigmas, muss die Versuchsperson vorher konditionierte Belohnungsreize ablehnen, um ein übergeordnetes Ziel zu erreichen. Durch den Vergleich zwischen an Schizophrenie erkrankten Patienten und gesunden Kontrollpersonen sollen mögliche neurofunktionelle Korrelate der Erkrankung identifiziert werden um die Entstehung der Symptomatik zu verstehen. Dies könnte in Zukunft die Möglichkeit bieten, gezielter in die Dysfunktion dieser Netzwerke einzugreifen, um Symptome der Patienten zu lindern und deren subjektives Leidensgefühl zu schmälern. 


\section{Material und Methoden}

\subsection{Stichprobe}

Im Rahmen dieser Studie wurden 16 Patienten mit der Diagnose Schizophrenie oder schizoaffektive Störung rekrutiert. Dabei handelte es sich um 14 männliche und zwei weibliche Versuchsteilnehmer im Alter von 18 bis 60 Jahren. Die Mehrzahl der Patienten befand sich zum Zeitpunkt der Untersuchung in stationärer Behandlung, ein Teil auch in tagesklinischer Behandlung der Klinik für Psychiatrie und Psychotherapie der Universitätsmedizin Göttingen. Voraussetzung zur Teilnahme war kein Vorliegen eines Ausschlusskriteriums. Einen Überblick der Einschluss- und Ausschlusskriterien bietet Tabelle 4.

Tabelle 4: Ein- und Ausschlusskriterien für Patienten.

\begin{tabular}{|c|c|}
\hline E inschlusskriterien & $\begin{array}{l}\text { - } \quad \text { Alter zwischen } 18 \text { und 60 Jahren } \\
\text { - Erkrankung des schizophrenen Formenkreises (F20/ F25) }\end{array}$ \\
\hline Ausschlusskniterien & $\begin{array}{l}\text { - } \text { nicht vorhandene Aufklärungs- oder Einwilligungsfähigkeit } \\
\text { - } \text { Klaute Suizidalität } \\
\text { - } \text { neurologische Erkrankungen } \\
\text { - } \text { Drogenkonsum im letzten Monat } \\
\text { - } \text { Substanzabhängigkeit, aktuell oder anamnestisch } \\
\text { - } \text { unkorrigierte Sehbehinderung } \\
\text { - } \text { schwere körperliche Beeinträchtigung } \\
\text { - } \text { Minderbegabung } \\
\text { - } \text { akute Eigen- oder Fremdgefährdung } \\
\text { - Kontraindikation zur MR-Untersuchung }\end{array}$ \\
\hline
\end{tabular}


Für die Kontrollgruppe wurden 16 gesunde Probanden rekrutiert, bei denen keine D iagnose einer psychiatrischen Erkrankung nach ICD-10 vorlag. Die Rekrutierung erfolgte größtenteils aus passenden Probanden einer Parallelstudie und aus wissenschaftlichen Mitarbeitem der Forschergruppen. Darüber hinaus wurden Kontrollprobanden über entsprechende Aushänge (Universität, öffentliche Aushänge, etc.) gesucht. Um eine gute Vergleichbarkeit zwischen den beiden Gruppen zu gewährleisten, wurden weit mehr Personen initial gesichtet, als dann tatsächlich als Kontrollpersonen rekrutiert wurden. Durch den hohen Aufwand in der Rekrutierungsphase ließ sich eine Gruppe von Kontrollpersonen zusammenstellen, die sowohl bezüglich Alter, Geschlecht als auch im Bildungsgrad dem der Patientenkohorte sehr gut entsprach. Die soziodemographischen D aten der Stichprobe sind in Tabelle 5 dargestellt.

Tabelle 5: Soziodemographische D aten der Stichprobe.

\begin{tabular}{|l|l|l|l|l|}
\hline & $\begin{array}{l}\text { Patienten } \\
(\mathbf{n = 1 6 )}\end{array}$ & $\begin{array}{l}\text { Kontrollen } \\
(\mathbf{n = 1 6 )}\end{array}$ & $\begin{array}{l}\text { F-Wert } \\
\mathbf{( d f} \mathbf{1})\end{array}$ & p-Wert \\
\hline $\begin{array}{l}\text { Alter } \\
\text { (Jahre; MW } \pm \text { SD) }\end{array}$ & $31,1 \pm 10,0$ & $30,8 \pm 9,9$ & 0,008 & 0,930 \\
\hline $\begin{array}{l}\text { Alter } \\
\text { (Jahre; Bereich) }\end{array}$ & $22-52$ & $21-58$ & - & - \\
\hline Geschlecht (m:w) & $14: 2$ & $14: 2$ & - & - \\
\hline $\begin{array}{l}\text { Bildungsdauer } \\
\text { (Jahre; MW } \pm \text { SD) }\end{array}$ & $14,6 \pm 2,0$ & $15,1 \pm 2,6$ & 0,368 & 0,548 \\
\hline
\end{tabular}

MW: Mittelwert; SD: Standardabweichung; m: männlich; w: weiblich

Alle Versuchsteilnehmer erhielten eine Aufwandsentschädigung in Form eines Mindestbetrages in Höhe von 30 Euro. Des Weiteren bestand die Möglichkeit, eine erfolgsabhängige Zulage zu bekommen, sodass jeder Versuchsteilnehmer bis zu 60 Euro erhalten konnte.

Alle Probanden wurden vor Durchführung der Untersuchung sowohl mündlich als auch schriftlich über die Studie informiert. Es wurde über den Inhalt, den Ablauf und die Ziele sowie insbesondere auch bezüglich möglicher Risiken aufgeklärt. Die Teilnahme erfolgte 
auf freiwilliger Basis. Eine Einverständniserklärung liegt von allen teilnehmenden Probanden vor. Alle erhobenen patientenbezogenen Daten wurden anonymisiert. Die Studie wurde durch die Ethik-Kommission der Universitätsmedizin Göttingen genehmigt (Antragsnummer 14/3/09).

\subsection{Psychopathologische Skalen}

Um die Patienten in Bezug auf die Schwere ihrer Erkrankung objektiv einordnen zu können, wurde anhand von verschiedenen Skalen der Grad ihrer Symptomatik erfasst. Noch am gleichen Tag der fMRT-Untersuchung wurden die Patienten in Bezug auf ihre Erkrankung bzw. ihre Symptomatik ausführlich befragt. Hierbei wurden die im Folgenden beschriebenen Skalen verwendet (siehe auch Anhang). Die Ergebnisse der Patienten sind in Tabelle 6 dargestellt.

\subsubsection{Positive and Negative Syndrome Scale (PANSS)}

Um die Patienten hinsichtlich ihrer Positiv- und Negativsymptomatik zu beurteilen, wurde die Positive and N egative Syndrom Scale (PANSS) erhoben. D iese Skala besteht aus einem 3040 minütigen formalisierten psychiatrischen Interview, in dem 30 Symptome (jeweils sieben positive und sieben negative Symptome sowie 16 allgemeine Symptome) anhand einer siebenstufigen Skala von 1 (nicht vorhanden) bis 7 (extrem ausgeprägt) bewertet werden. Die PANSS-Beurteilung basiert auf der Befindlichkeit des Patienten in den letzten sieben Tagen.

Die in der Studie eingeschlossenen Patienten haben mittlere PANSS-Werte im unteren Bereich und zeigen damit eine nur milde Ausprägung.

\subsubsection{Clinical Global Impression Scale (CGI)}

D ie Clinical G lobal Impression Scale (CGI) ist eine international in der Psychiatrie verwendete Skala zur allgemeinen und diagnoseübergreifenden Bestimmung des aktuellen Schweregrads einer Erkrankung. Hier bewertet der Untersucher die Ausprägung der Erkrankung des Patienten anhand einer Skala von 1 bis 7 Punkten. Je mehr Punkte dem Patienten zugeteilt werden, desto stärker ist seine Symptomatik ausgeprägt.

D ie in die Studie eingeschlossenen Patienten haben einen mittleren CGI-Wert von 4 und sind damit im D urchscnitt als moderat krank einzustufen. 


\subsubsection{Montgomery-Asberg Depression Scale (MADRS)}

Um depressive Symptome des Patienten zu erfassen, bedienten wir uns der MontgomeryA sberg D epression Scale (MADRS). Hierbei handelt es sich um eine Beurteilungsskala zur Einschätzung depressiver Symptomatik. Sie enthält insgesamt zehn Elemente, die jeweils einem Symptom entsprechen, und durch den Untersucher auf der Grundlage eines Interviews und von Beobachtungen erfasst werden. Die Items erfassen im Einzelnen die folgenden Bereiche: sichtbare Traurigkeit, berichtete Traurigkeit, innere Spannung, Schlaflosigkeit, Appetitverlust, Konzentrationsschwierigkeiten, Untätigkeit, G efühllosigkeit, pessimistische Gedanken, Selbstmordgedanken. Die Unterteilung der einzelnen Elemente erfolgt von den Stufen 0 bis 6 . Je höher die Stufe, desto stärker die depressive Symptomatik.

Die in der Studie eingeschlossenen Patienten haben einen mittleren MADRS-Wert von 11,8 und zeigen somit eine milde Depression.

Tabelle 6: Psychopathologische Skalen der Patienten $(n=16)$.

\begin{tabular}{|l|l|l|}
\hline & Mittelwert & Standardabweichung \\
\hline PANSS positiv & 11,4 & 3,4 \\
\hline PANSS negativ & 12,2 & 4,5 \\
\hline PANSS allgemein & 27,2 & 5,1 \\
\hline CG I & 4,0 & 0,8 \\
\hline MADRS & 11,8 & 6,4 \\
\hline
\end{tabular}

CGI: Clinical G lobal Impression Scale; MADRS: Montgomery-A sberg Depression Rating Scale; PANSS: Positive and N exative Syndrome Scale

\subsection{Medikation}

D ie Medikation der Patienten ist in Tabelle 7 dargestellt. Alle Patienten befanden sich zum Zeitpunkt der Datenerhebung entweder in stationärer oder ambulanter Behandlung. Somit standen alle Patienten unter verschiedener antipsychotischer, antidepressiver oder akut dämpfender Medikation. Das Vorhandensein einer Medikation kann im Allgemeinen einen 
limitierenden Faktor im Bezug auf die Interpretierbarkeit der erhobenen D aten darstellen. Hierauf wird in der D iskussion der Ergebnisse näher eingegangen.

Tabelle 7: Medikation der Patienten.

\begin{tabular}{|l|l|l|}
\hline & $\begin{array}{l}\text { Anzahl } \\
(\mathbf{n = 1 6 )}\end{array}$ & $\begin{array}{l}\text { Anteil } \\
\mathbf{( \% )}\end{array}$ \\
\hline Antipsychotika (atypisch) & 16 & 100 \\
\hline Antipsychotika (typisch) & 3 & 18,8 \\
\hline Antidepressiva & 7 & 43,8 \\
\hline Anticholinergika & 3 & 18,8 \\
\hline B-Blocker & 3 & 18,8 \\
\hline Benzodiazepine & 1 & 6,3 \\
\hline
\end{tabular}

\subsection{Magnetresonanztomografie (MRT)}

D ie Magnetresonanztomografie (MRT) ist ein bildgebendes Verfahren, das insbesondere in der medizinischen Diagnostik zum Einsatz kommt. Es dient der Darstellung von Strukturen und Organen und deren Funktion. Die MRT basiert auf der physikalischen Eigenschaft von sehr starken Magnetfeldern sowie elektromagnetischen Wechselfeldern im Radiofrequenzbereich. D er zugrunde liegende Mechanismus basiert auf der Tatsache, dass A tomkerne, wie etwa Wasserstoffkerne, in untersuchten Geweben gezielt (phasensynchron und resonant) elektromagnetisch angeregt werden und dann bei der Rückkehr in ihren Ursprungszustand ein spezifisches Signal abgeben. D ieses ist sowohl von der molekularen Umgebung als auch von den chemischen Eigenschaften abhängig, in der sich der angeregte Wasserstoffkern befindet. Das hat zur Folge, dass sich Gewebearten charakteristisch in ihrem Signal unterscheiden, was zu verschiedenen Signalstärken (Helligkeiten) im entstandenen Bild führt (Huettel et al. 2014). 


\subsubsection{Funktionelle Magnetresonanztomografie (fMRT)}

Ein spezielles Verfahren der MRT zur nicht-invasiven Charakterisierung von Hirnfunktionsänderungen ist die fMRT. Diese bildet die zentrale Untersuchungsmethode der vorliegenden Studie und wird im folgenden Abschnitt kurz erläutert (vergleiche auch (D erntl et al. 2010; Huettel et al. 2014)).

Mittels der fMRT werden Veränderungen in der G ewebedurchblutung in verschiedenen Hirnarealen gemessen, die durch den veränderten Energiebedarf aktivierter Nervenzellen hervorgerufen werden. Dadurch ist die Darstellung von funktionellen Abläufen im $\mathrm{G}$ ehirngewebe möglich. Bei diesen Messungen ist die lokale Oxygenierung des Blutes der entscheidende, die Signalintensität beeinflussende Parameter. Grundlage für die fMRT ist der sogenannte BOLD (Blood Oxygenation L evel D ependent) Effekt. Hierbei macht man sich die unterschiedlichen magnetischen Eigenschaften von oxygeniertem und desoxygeniertem Blut zunutze. Verantwortlich dafür ist das an Hämoglobin gebundene Eisen. Dieses wird für den Sauerstofftransport oxygeniert, es entsteht Oxyhämoglobin. Oxygeniertes Hämoglobin ist diamagnetisch und hat keinen Einfluss auf die magnetischen Eigenschaften des umgebenden G ewebes. D esoxygeniertes Hämoglobin hingegen ist paramagnetisch. Es ist in seiner Eigenschaft wesentlich stärker magnetisierbar. Dies führt zu darstellbaren Magnetfeldveränderungen.

Bei lokalen Aktivitätssteigerungen kommt es zu einem erhöhten Metabolismus, welcher sich regional in einem erhöhten zerebralen Blutfluss widerspiegelt. Das Verhältnis von oxygeniertem zu desoxygeniertem Hämoglobin ändert sich. Desoxyhämoglobin wird aus dem Gewebe ausgewaschen, was zu einem Signalanstieg führt. In der fMRT bedeutet dies, dass sich in den BOLD-sensitiven Bildern ein stärkeres MR-Signal in den Bereichen zeigt, in denen das Blut stärker oxygeniert ist.

Führt man am gleichen Objekt Aufnahmen zu verschiedenen Zeitpunkten durch (Ruhezustand vs. stimulierter Zustand), können diese durch statistische Analyseverfahren miteinander verglichen und Hirnaktivitätsänderungen räumlich zugeordnet werden.

Eine Aufstellung der in der Studie verwendeten MR-Sequenzen und eingestellten Messparameter findet sich im Anhang. 


\subsection{Paradigma}

\subsubsection{Zielsetzung}

Zum Einsatz kam das in unserer Arbeitsgruppe entwickelte D esire Reason-D ilemma (D RD)Paradigma (Diekhof und Gruber 2010). Hierbei dürfen die Versuchspersonen in unterschiedlichen Phasen des Paradigmas zuvor konditionierte Reize als Bonus annehmen (Bonusverfolgung) oder müssen diese ablehnen, um ein übergeordnetes Ziel zu erreichen (Zielverfolgung). Dadurch sollen zum einen die Hirnareale des dopaminergen Belohnungssystems sowie Regionen, die im Rahmen von Entscheidungsprozessen involviert sind, aktiviert werden und zusätzlich das veränderte Aktivitätsmuster bei Ablehnung konditionierter Bonusreize in der Zielverfolgung dargestellt werden.

\subsubsection{Durchfühnung}

Am Tag vor der eigentlichen fMRT-Untersuchung wurde in einer Trainingssitzung zunächst eine operante Konditionierungsaufgabe, gefolgt von einem Training der experimentellen Verhaltensaufgabe, in einem dafür vorgesehenen Trainingsraum durchgeführt. Es wurde sichergestellt, dass sämtliche Teilnehmer unter gleichen, standardisierten Bedingungen trainiert wurden. Es wurde für ein störungsfreies Umfeld gesorgt, in welchem sich die Probanden voll und ganz ihrer Aufgabe widmen konnten. Nach ausführlicher Aufklärung über den Ablauf des Verhaltensexperimentes bearbeiteten die Teilnehmer die experimentelle Aufgabe an einem handelsüblichen Computer.

Am Tag der fMRT-Untersuchung wurde den Teilnehmern die experimentelle Verhaltensaufgabe nochmals erläutert, bevor sie im MR-Tomografen positioniert wurden und dort die Aufgabe während der fMRT durchführten. Es wurden zwei D urchgänge der Aufgabe durchgeführt, wobei innerhalb eines Durchgangs nach zwei Aufgabenblöcken einer Sorte der Aufgabenblock wechselte. Jeder Durchgang begann stets mit zwei Aufgabenblöcken „Bonusverfolgung“, denen zwei Aufgabenblöcke „Zielverfolgung“ folgten. Es kamen über die zwei D urchgänge insgesamt 40 Aufgabenblöcke zum Einsatz, welche aus je 20 Aufgabenblöcken „Bonusverfolgung” und 20 Aufgabenblöcken "Zielverfolgung" bestanden. Wie viele Q uadrate in einer Sequenz erschienen, war von den Antworten und der Schnelligkeit des Probanden abhängig. 


\subsubsection{Operante Konditionierung}

Im Rahmen der operanten Konditionierung sah der Proband auf dem Monitor nacheinander erscheinende farbige Q uadrate (z. B. rot, grün, blau, gelb, rosa etc.). Mittels Tastendrucks auf der Tastatur des Computers sollte der Proband die einzelnen farbigen Q uadrate entweder annehmen oder ablehnen. Mit Tastendruck des rechten Zeigefingers (linke Taste) wurde ein Q uadrat beziehungsweise die Farbe angenommen. Mit Tastendruck des rechten Mittelfingers (rechte Taste) wurde eine Farbe abgelehnt. Dies geschah ohne Zeitvorgabe. In den Q uadraten erschienen, je nach Tastendruck, entsprechende Zahlen im Wert von -10, +10 oder 0. Der Proband bemerkte früher oder später, dass es bei den meisten Farben, keine Rolle spielt, ob er sie annimmt oder ablehnt, da bei den meisten Farben eine 0 erschien. Er bemerkte jedoch auch, dass die Farben Rot und Grün, wenn sie angenommen wurden, 10 Bonuspunkte einbrachten. Er stellte also einen Zusammenhang zwischen der Farbe der Quadrate und der damit einhergehenden Belohnung her. Wichtig war bei dieser Vorbereitung für die eigentliche Aufgabe, dass die operante Konditionierung ausreichend lange durchgeführt wurde, damit die Konditionierungseffekte im dopaminergen System sichergestellt werden konnten.

\subsubsection{Experimentelle Verhaltensaufgabe}

Vor Durchführung der eigentlichen Verhaltensaufgaben wurden diese den Probanden genau erklärt. Sie wurden instruiert, so rasch wie möglich und so genau wie möglich zu antworten. Die Hauptaufgabe in diesem Verhaltensparadigma ist es, so viele Punkte wie möglich zu sammeln, um eine hohe Gesamtpunktzahl zu erreichen. Je höher die Gesamtpunktzahl am Ende ist, desto höher ist auch die Aufwandsentschädigung, welche der Proband nach Abschluss der Untersuchung erhält. Wie bereits erwähnt, gab es für jeden Versuchsteilnehmer grundsätzlich eine Aufwandsentschädigung von 30 Euro. Zusätzlich konnte jeder Proband, entsprechend seiner Leistung im Verhaltensparadigma, bis zu 30 Euro dazu gewinnen.

$\mathrm{Zu}$ Beginn der Aufgabe wurde der Versuchsperson auf dem Bildschirm ein Kreuz in der Mitte präsentiert, welches sie fixieren sollte. Der Ablauf des Paradigmas wurde in 2 Aufgabenblöcke unterteilt. Es wurde unterschieden zwischen einem Aufgabenblock „Zielverfolgung“ (Abbildung 2) und einem Aufgabenblock „Bonusverfolgung“ (Abbildung 3). Dem Probanden wurde zu Beginn jedes Aufgabenblocks auf dem Monitor durch ein großes Z für „Zielverfolgung“ und ein großes B für „Bonusverfolgung“ angezeigt, welcher 
Aufgabenblock als nächstes folgt. Anschließend erschienen zwei Quadrate mit unterschiedlichen Farben. Diese stellten die unmittelbaren Zielreize dar und mussten vom Proband gut einprägt werden. Der Proband musste sich also zum einen die zwei gezeigten Farben merken und zum anderen auch die ganze Zeit wissen, ob er sich in der Zielverfolgung oder in der Bonusverfolgung befindet.

In den Einzelaufgaben innerhalb eines Aufgabenblocks erschienen nun einzelne farbige Q uadrate und der Proband musste sich entscheiden, ob er dieses Q uadrat annimmt oder ablehnt. Befand sich der Proband in der Zielverfolgung, so sollte er alle Quadrate annehmen, die den beiden vorher gezeigten Farben entsprachen. Alle anderen Farben sollten abgelehnt werden. Nach jedem erfolgreich durchgeführten Aufgabenblock bekam der Proband Punkte. Befand sich der Proband jedoch in der Bonusverfolgung, so sollte er alle Q uadrate annehmen, die den zwei vorher gezeigten Farben entsprachen und zusätzlich Quadrate der Farben Rot und Grün (die vorher konditionierten Bonusreize), welche Bonuspunkte einbrachten. Nur in der Bonusverfolgung war es möglich Bonuspunkte zu bekommen. Der Proband musste also stets wissen, in welchem Aufgabenblock er sich befand und welche Farben relevant für den aktuellen Aufgabenblock waren. Entschied sich der Proband falsch oder antwortete er nicht in dem vorgegebenen Zeitfenster (900 ms), so wurden ihm die Punkte aus diesem Aufgabenblock nicht gutgeschrieben. Der Aufgabenblock brach ab und es wurde mit dem nächsten Aufgabenblock fortgefahren. Nur wenn der Proband die Q uadrate in einem kompletten Aufgabenblock korrekt annahm bzw. ablehnte, galt dieser als vollständig, und er bekam die gesammelten Punkte. 


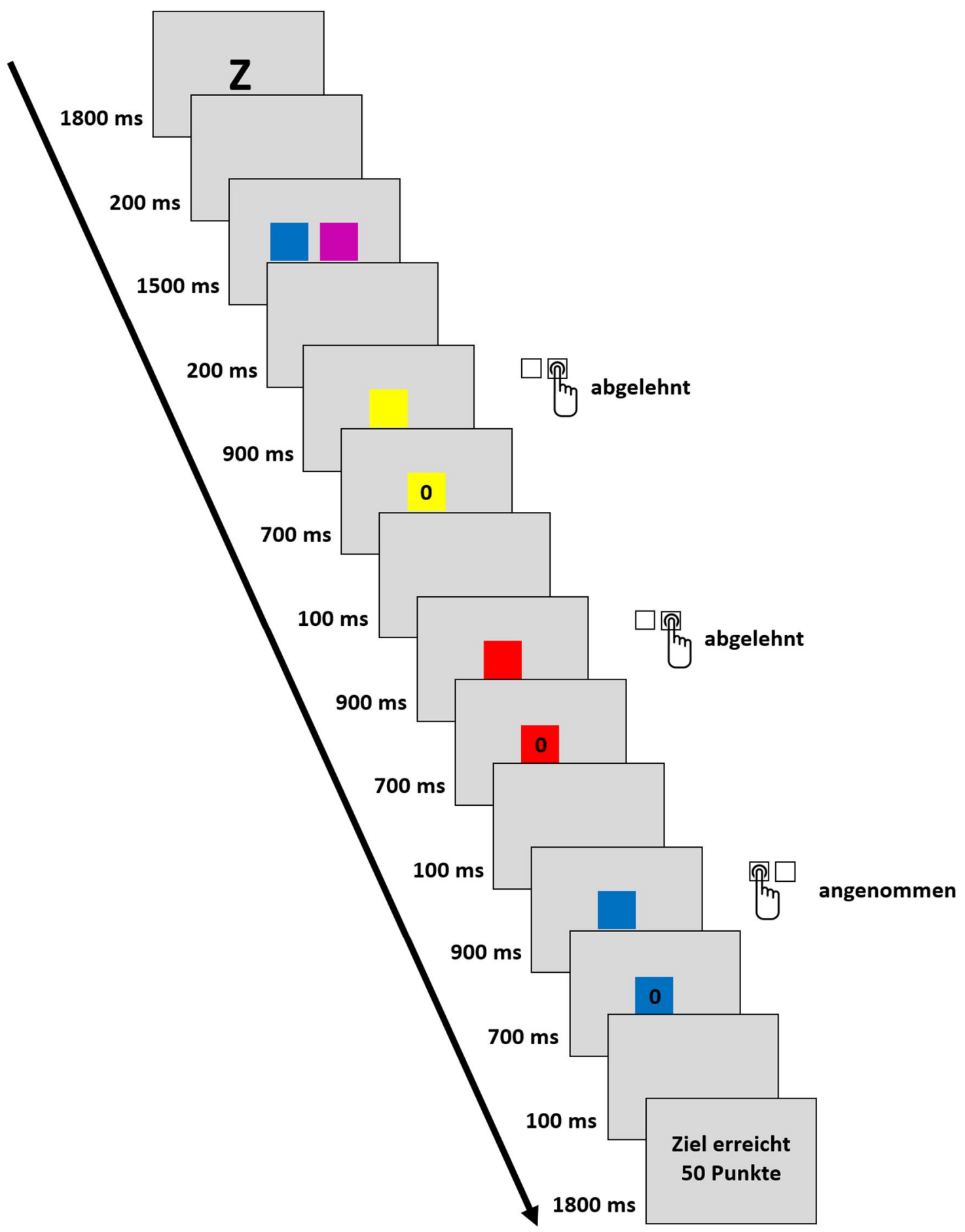

Abbildung 2: Paradigma: Schematischer Ablauf eines Aufgabenblocks „Zielverfolgung“. Die Testperson sollte nur die beiden zu Anfang gezeigten Farben (hier: blau, violett) annehmen. Bonusfarben (rot, grün) mussten abgelehnt werden. Nähere Erläuterungen im Text. Verändert aus D iekhof und Gruber (2010). 




Abbildung 3: Paradigma: Schematischer Ablauf eines Aufgabenblocks „Bonusverfolgung“. Die Testperson sollte die beiden zu Anfang gezeigten Farben (hier: blau, violett) annehmen. Zusätzlich durften Bonusfarben (rot, grün) angenommen werden. Nähere Erläuterungen im Text. Verändert aus D iekhof und Gruber (2010). 


\subsection{3 fMRT-Untersuchung}

D as Verhaltensparadigma wurde mit der Software Presentation (Neurobehavioral Systems, Albany, CA, USA), sowohl auf dem Computer im Trainingsraum als auch in der anschließenden fMRT-Untersuchung durchgeführt. Die fMRT-Untersuchung erfolgte bei allen teilnehmenden Probanden am Tag nach dem Training im MR-Tomografen der Wissenschaftlichen Serviceeinrichtung MR-Forschung der Universitätsmedizin Göttingen (3 Tesla MR-System, Siemens Magnetom Trio, Erlangen, D eutschland).

Die Probanden legten sich auf die Untersuchungsliege und wurden mit einer MRtauglichen LCD-Brille (Resonance Technology, Northridge, CA, USA) ausgestattet, in der die Probanden den bereits bekannten Bildschirm aus den Trainingsübungen vor sich sahen. Um das Paradigma bearbeiten zu können, bekamen sie eine Tastbox (Current Designs, Philadelphia, PA, USA) in die rechte Hand. Diese bestand aus zwei Knöpfen, welche genau wie im Training gedrückt werden konnten: der linke Knopf für den Zeigefinger und der rechte Knopf für den Mittelfinger der rechten Hand.

Über die gesamte Dauer der Untersuchung hinweg bestand für den Probanden die Möglichkeit, bei auftauchenden Schwierigkeiten oder Fragen, über eine Sprechanlage mit dem Personal zu kommunizieren. Zusätzlich verfügte der Proband über einen Notfallknopf, mit dem er jederzeit einen gewünschten Abbruch der Untersuchung anzeigen konnte. Um den Probanden möglichst bequem zu lagern und auch vor der relativ hohen Lärmbelastung während der fMRT-Untersuchung zu schützen, wurden sowohl ein Gehörschutz als auch Schaumstoffpolster verwendet. Nach optimaler Vorbereitung des Probanden konnte mit der Messung begonnen werden.

Vor der eigentlichen funktionellen MR-Untersuchung wurde eine anatomische Aufnahme des Kopfes des Probanden angefertigt. Diese Aufnahme diente zum einen dazu das Messvolumen für die fMRT-Untersuchung zu definieren und wurde zum anderen im Rahmen der Datenanalyse zur Koregistrierung der funktionellen Daten mit der individuellen Hirnanatomie des Probanden verwendet. D anach folgte auf dem Bildschirm nochmals eine kurze Erläuterung über den Inhalt der Verhaltensaufgabe und das D rücken der Tasten. Wenn der Proband bereit war, die kommenden Aufgabenblöcke zu bearbeiten, wurde mit der funktionellen Messung begonnen.

Das Verhaltensparadigma dauerte pro Durchgang jeweils sechs Minuten. Da zwei D urchgänge bearbeitet wurden, betrug die Gesamtdauer also 12 Minuten. Über dieses Verhaltensparadigma hinaus wurden im Rahmen der Studie noch weitere fMRT- 
Untersuchungen durchgeführt, nämlich eine Oddball-A ufgabe (Wolter et al. 2016) und eine Messung der Ruheaktivität des Gehirns. Diese Untersuchungen waren jedoch nicht Gegenstand der vorliegenden Arbeit und werden hier nicht weiter betrachtet. Für die Gesamtdauer der Untersuchung am Tag der Messung wurden 1,5 Stunden vorgesehen, wobei die reine Messzeit im MR-Tomografen etwa 50 Minuten in Anspruch nahm.

\subsection{Datenanalyse}

\subsubsection{Analyse der Performanzdaten}

Die statistische Analyse der Verhaltensdaten erfolgte über die Software SPSS (IBM, Armonk, NY, USA). Mittels einer ANOVA (A nalysis of $\mathrm{V}$ arianoe) und anschließendem post hoc t-Test wurden Unterschiede in den Performanzparametern (Trefferquote, Fehlerquote, angenommene Boni) zwischen den beiden Gruppen (Patienten, Kontrollpersonen) analysiert. Es wurde eine Signifikanzschwelle von $p<0,05$ verwendet.

\subsubsection{Analyse der fMRT-Daten}

Die Analyse der fMRT-D aten wurde mit der Software SPM5 (Wellcome D epartment of Cognitive Neurology, University College London, London, UK) durchgeführt. Zunächst wurden die Daten importiert und aus dem Rohdatenformat des MR-Tomografen (dicom) in das ANALYZE-Format (img, hdr) konvertiert. D anach wurden die Dateien mithilfe des Programms Rename, entsprechend der internen Nomenklatur der Arbeitsgruppe, umbenannt.

Die Vorverarbeitung der Daten orientierte sich an üblichen Standardverfahren im Zusammenhang mit fMRT-D atenanalysen. Sie beinhaltete eine Korrektur für räumliche Verzerrungen, die zeitliche Normalisierung (da eine Aufnahme des gesamten Gehirns über einen Zeitraum von 1,8 Sekunden verteilt geschieht, werden die zu unterschiedlichen Zeitpunkten aufgenommenen D aten auf einen Zeitpunkt innerhalb des Aufnahmeintervalls normalisiert), die räumliche Glättung sowie die räumliche Normalisierung der D aten.

Die Datenanalyse wurde auf Grundlage des allgemeinen linearen Modells (GLM) durchgeführt. Es wurden insgesamt 11 Regressoren erstellt. Für jeden der beiden Aufgabenblöcke (Bonusverfolgung und Zielverfolgung) wurden drei Regressoren erstellt, welche die Darbietung von Zielreizen, Bonusreizen und neutralen Reizen repräsentierten (insgesamt sechs Regressoren). Ferner wurden die anderen Phasen des 
Verhaltensparadigmas ebenfalls modelliert. Diese waren zwar nicht von Interesse für die übergeordnete Fragestellung, dennoch mussten sie in die Analyse integriert werden, um entsprechenden Effekten Rechnung zu tragen. Es handelte sich dabei um die Hinweise zu Anfang eines Aufgabenblocks, ob es sich um eine Bonusverfolgung oder Zielverfolgung handelte (zwei Regressoren). Die nachfolgende Präsentation der beiden Zielfarben in jedem Aufgabenblock wurde durch einen Regressor für beide Aufgabenblöcke repräsentiert. Ferner wurde die Rückmeldung am Ende eines erfolgreich absolvierten Aufgabenblockes (ein Regressor) sowie die Rückmeldung über einen abgebrochenen Aufgabenblock (ein Regressor) modelliert. Um die zeitlichen Charakteristika der hämodynamischen Antwortfunktion zu berücksichtigen, wurden die Regressoren entsprechend gefaltet, sodass die Regressoren den zu erwartenden Signaländerungen in den fMRT-D aten entsprachen.

Im Rahmen der statistischen Analyse wurden verschiedene Kontraste erstellt, zunächst innerhalb der jeweiligen G ruppe, d. h. einmal nur für die Patienten und einmal nur für die Kontrollpersonen. Im nächsten Schritt wurde ein Vergleich zwischen den Gruppen (Gruppenanalyse) durchgeführt, um signifikante Unterschiede zwischen der Patientengruppe und der Kontrollgruppe darzustellen. Aus den Aktivierungskarten wurden die Aktivierungscluster mit den jeweiligen Koordinaten und dem t-Wert extrahiert. Für die erstellten Aktivierungskarten wurde eine Signifikanzschwelle von $\mathrm{p}<0,005$ zugrundegelegt. Um den multiplen statistischen Vergleichen Rechnung zu tragen, wurde eine Korrektur auf G rundlage der F alse D iscovery Rate mit einer Signifikanzschwelle von $\mathrm{p}<$ 0,05 angewendet. Für Hirnregionen, für die unter Berücksichtigung der vorangegangenen Studien aus unserer Arbeitsgruppe (Diekhof und Gruber 2010; Diekhof et al. 2012a; Diekhof et al. 2012c) eine a priori-Hypothese vorlag (vStr, VTA) wurde das Verfahren der Korrektur für kleine Volumina (SVC) eingesetzt.

Mithilfe der psychophysiologischen Interaktionsanalyse (PPI) (Friston et al. 1997) ist man in der Lage, funktionelle Interaktionen zwischen Hirnregionen in Abhängigkeit von der gerade durchgeführten Aufgabe zu erfassen. Eine anschauliche Erklärung der Methode sowie ein Vergleich $\mathrm{zu}$ anderen Verfahren der Charakterisierung funktioneller Konnektivität ist bei O 'Reilly et al. (2012) zu finden. In der vorliegenden Studie wurde die PPI eingesetzt, um solche funktionellen Interaktionen zwischen dem vStr und präfrontalen Hirnarealen während der Dilemmasituation, wenn also zuvor konditionierte Belohnungsreize abgelehnt werden mussten, um das übergeordnete Ziel zu erreichen, zu charakterisieren. Vorherige Ergebnisse aus unserer Arbeitsgruppe zeigten einen 
inhibitorischen Einfluss des avPFC auf das vStr bei gesunden Versuchspersonen während der Phase der Zielverfolgung im vorliegenden Paradigma (Diekhof und Gruber 2010; Diekhof et al. 2012a; Diekhof et al. 2012c). Entsprechend wurde das bilaterale vStr als Startregionen für die PPI-Analyse gewählt. Aus diesen Regionen wurde der Signalzeitverlauf (physiologischer Vektor) extrahiert. Der Interaktionskontrast der mit Belohnungsreizen assoziierten Aktivität in der Bonusverfolgung gegenüber der Zielverfolgung diente als psychophysiologischer Vektor. Mithilfe der PPI-Analyse konnte nun die Änderung der funktionellen Konnektivität, ausgehend von den Startregionen, unabhängig von den Haupteffekten der Aktivitätsänderungen aufgrund der Verhaltensaufgabe ermittelt werden. Zum G ruppenvergleich kam eine Signifikanzschwelle von $p<0,001$ zum Einsatz. Auch hier wurde für Regionen mit einer a priori-Hypothese aufgrund von vorherigen Studien aus unserer Arbeitsgruppe (D iekhof und G ruber 2010; D iekhof et al. 2011) das Verfahren der Korrektur für kleine Volumina eingesetzt. 


\section{Ergebnisse}

\subsection{Penformanzdaten}

Es zeigten sich signifikante Haupteffekte für die Faktoren Gruppe $(F(1,30)=23,9$, $p<$ $0,001)$ und Aufgabe $(F(1,30)=94,2, p<0,001)$ sowie ein signifikanter Interaktionseffekt $(F(1,30)=8,3, p<0,01)$. Im Vergleich zwischen Patienten und Kontrollpersonen zeigten die Patienten signifikant schlechtere Leistungen bzgl. der prozentualen Trefferquote $\mathrm{t}(30)$ $=4,9, \mathrm{p}<0,001$; Tabelle 8).

\subsubsection{Desire-Kontext}

Post hoc t-Tests zeigten eine signifikant schlechtere Performanz der Patienten, wenn es darum ging, den vorher konditionierten Belohnungsreiz in der Bonusverfolgung (D esireKontext) anzunehmen $(\mathrm{t}(30)=4,3, \mathrm{p}<0,001)$. Auch war die Performanz hinsichtlich anzunehmender Zielreize signifikant schlechter $(t(30)=2,4, p=0,02$; Tabelle 8).

\subsubsection{Reason-Kontext}

Post hoc t-Tests zeigten auch in der Zielverfolgung (Reason-Kontext) für die Patienten eine signifikant schlechtere Performanz im Vergleich zu Kontrollpersonen. So zeigte sich in der G ruppe der Patienten eine signifikant schlechtere Leistung, wenn es darum ging, in der Zielverfolgung den angebotenen Bonus, welcher abgelehnt werden musste, um den Aufgabenblock „Zielverfolgung" erfolgreich abzuschließen, abzulehnen $(t(30)=2,4, p=$ 0,02). Wie auch schon in Zusammenhang mit der Bonusverfolgung war auch in der Zielverfolgung die Performanz hinsichtlich anzunehmender Zielreize signifikant schlechter $(\mathrm{t}(30)=4,6, \mathrm{p}<0,001$; Tabelle 8).

Zusammenfassend zeigte sich also eine durchweg weniger gute Performanz bei den Patienten, sowohl im Aufgabenblock „Bonusverfolgung“ als auch im Aufgabenblock „Zielverfolgung". 
Tabelle 8: Verhaltensdaten der Stichprobe.

\begin{tabular}{|l|l|l|l|}
\hline & $\begin{array}{l}\text { Patienten } \\
(\mathbf{n = 1 6 )}\end{array}$ & $\begin{array}{l}\text { Kontrollen } \\
(\mathbf{n = 1 6 )}\end{array}$ & p-Wert \\
\hline G enauigkeit insgesamt (\%) & $83,1 \pm 5,0$ & $91,4 \pm 4,6$ & $<0,001$ \\
\hline Bonusverfolgung & & & \\
\hline Boni (korrekt angenommen; \%) & $63,8 \pm 16,6$ & $85,5 \pm 11,5$ & $<0,001$ \\
\hline Ziele (korrekt angenommen; \%) & $89,8 \pm 5,9$ & $94,3 \pm 4,4$ & 0,021 \\
\hline Zielverfolgung & & & \\
\hline Boni (korrekt abgelehnt; \%) & $88,0 \pm 10,7$ & $95,2 \pm 4,9$ & 0,024 \\
\hline Ziele (korrekt angenommen; \%) & $90,7 \pm 4,8$ & $97,0 \pm 2,6$ & $<0,001$ \\
\hline
\end{tabular}

Angegeben ist jeweils der Mittelwert in Prozent \pm Standardabweichung

\section{2 fMRT-Daten}

\subsubsection{Desine-Kontext}

In dieser experimentellen Aufgabe zeigten sich im Einklang mit vorherigen Studien aus unserer Arbeitsgruppe (Diekhof und Gruber 2010; Diekhof et al. 2012a; Diekhof et al. 2012b) in beiden Gruppen belohnungsbezogene Aktivierungen im bilateralen vStr und in der VTA. Darüber hinaus zeigten sich ebenfalls kortikale Aktivierungen in präfrontalen, orbitofrontalen und parietalen Regionen. So zeigten sich unter anderem Bereiche des bilateralen avPFC, des bilateralen mittleren frontalen Gyrus, des bilateralen inferioren frontalen Verbindungsareals (ein Areal im Bereich des Aufeinandertreffens des inferioren frontalen Sulkus und des präzentralen Sulkus), der bilateralen frontalen Augenfelder, des frontoopercularen Kortex und im intraparietalen Kortex aktiviert (Tabelle 9). 
Tabelle 9: Aktivierungen der Patienten und Kontrollen im Aufgabenblock „Bonusverfolgung" (D esireKontext).

\begin{tabular}{|l|l|l|l|l|}
\hline & \multicolumn{2}{|l|}{ Patienten } & \multicolumn{2}{l|}{ Kontrollen } \\
\hline H imregion & $\begin{array}{l}\text { Koordinate } \\
(\mathrm{x}, \mathrm{y}, \mathrm{z} ; \mathrm{mm})\end{array}$ & $\mathrm{t}$-Wert & $\begin{array}{l}\text { Koordinate } \\
(\mathrm{x}, \mathrm{y}, \mathrm{z} ; \mathrm{mm})\end{array}$ & t-Wert \\
\hline L dorsales/ ventrales Striatum & -1269 & 4,8 & -1566 & 4,3 \\
\hline R dorsales/ ventrales Striatum & 1203 & 5,9 & 1263 & 6,0 \\
\hline L ventrale tegmentale Area & $-9-18-15$ & 4,1 & $-6-18-18$ & 5,3 \\
\hline R ventrale tegmentale Area & $6-24-18$ & 4,0 & $6-24-18$ & 6,2 \\
\hline $\begin{array}{l}\text { L antero-ventraler präfrontaler } \\
\text { Kortex }\end{array}$ & -304212 & 5,7 & -364212 & 4,5 \\
\hline $\begin{array}{l}\text { R antero-ventraler präfrontaler } \\
\text { Kortex }\end{array}$ & 364212 & 4,5 & 27450 & 4,6 \\
\hline $\begin{array}{l}\text { L anteriorer mittlerer frontaler } \\
\text { G yrus }\end{array}$ & -365121 & 3,6 & -365121 & 5,8 \\
\hline L mittlerer frontaler Gyrus & -333921 & 6,7 & -333921 & 3,9 \\
\hline R mittlerer frontaler Gyrus & 393333 & 5,2 & 363030 & 5,6 \\
\hline R inferiorer frontaler Sulkus & 333618 & 5,7 & 363921 & 4,7 \\
\hline $\begin{array}{l}\text { L inferiores frontales } \\
\text { Verbindungsareal/ posteriorer } \\
\text { inferiorer frontaler Gyrus }\end{array}$ & -42333 & 6,3 & -42333 & 3,7 \\
\hline $\begin{array}{l}\text { R inferiores frontales } \\
\text { Verbindungsareal/ posteriorer } \\
\text { inferiorer frontaler Gyrus }\end{array}$ & 51630 & 5,7 & 42342 & 5,3 \\
\hline L frontales Augenfeld & $-36-1563-651$ & 6,5 & $-36-1563$ & 2,4 \\
\hline R frontales Augenfeld & 6,3 & 3,948 \\
\hline
\end{tabular}




\begin{tabular}{|c|c|c|c|c|}
\hline $\mathrm{R}$ mittlerer frontaler Gyrus & 33654 & 3,5 & 33654 & 4,9 \\
\hline $\begin{array}{l}\text { L frontooperkularer Kortex/ } \\
\text { anteriore Insula }\end{array}$ & -30210 & 6,8 & -30183 & 6,1 \\
\hline $\begin{array}{l}\text { R frontooperkularer Kortex/ } \\
\text { anteriore Insula }\end{array}$ & 33213 & 7,8 & 33213 & 7,8 \\
\hline L intraparietaler Kortex & $-27-5742$ & 6,3 & $-30-5145$ & 6,0 \\
\hline $\mathrm{R}$ intraparietaler Kortex & $33-5442$ & 7,0 & $33-5445$ & 6,3 \\
\hline L intraparietaler Kortex & $-36-4545$ & 6,7 & $-39-4863$ & 4,0 \\
\hline L inferiorer parietaler Lobus & $-51-2745$ & 5,8 & $-45-3357$ & 3,8 \\
\hline $\mathrm{R}$ inferiorer parietaler Lobus & $54-3948$ & 6,9 & $45-3042$ & 6,5 \\
\hline $\begin{array}{l}\text { L posteriorer frontomedianer } \\
\text { Kortex }\end{array}$ & -61245 & 7,2 & -61251 & 8,2 \\
\hline $\begin{array}{l}\mathrm{R} \text { posteriorer frontomedianer } \\
\text { Kortex }\end{array}$ & 61545 & 7,6 & 271542 & 3,3 \\
\hline $\begin{array}{l}\text { L dorsaler anteriorer cingulärer } \\
\text { Kortex }\end{array}$ & -3933 & 5,3 & -3330 & 4,2 \\
\hline R mittlerer temporaler Gyrus & $54-39-12$ & 3,1 & $54-36-18$ & 4,5 \\
\hline L okzipitaler K ortex & $-15-99-6$ & 7,2 & $-15-93-12$ & 7,0 \\
\hline R okzipitaler Kortex & $21-969$ & 6,4 & $27-90-9$ & 6,6 \\
\hline L Cerebellum & $-36-57-24$ & 5,5 & $-33-51-24$ & 3,8 \\
\hline R Cerebellum & $33-48-24$ & 6,8 & $27-51-24$ & 6,1 \\
\hline L Thalamus & $-12-159$ & 4,4 & $-9-126$ & 3,8 \\
\hline R Thalamus & $18-39$ & 5,7 & $15-69$ & 5,9 \\
\hline
\end{tabular}




\begin{tabular}{|l|l|l|l|l|}
\hline $\begin{array}{l}\text { R Hippocampus/ } \\
\text { parahippocampaler Gyrus }\end{array}$ & - & - & $24-30-6$ & 2,7 \\
\hline
\end{tabular}

L: links; R: rechts

Im G ruppenvergleich (Tabelle 10) zeigte sich sowohl eine signifikante Hyperaktivierung bei Patienten (Patienten > Kontrollen; Abbildung 4) im linken vStr als auch eine Hyperaktivierung in verschiedenen frontalen und parietalen Regionen, wie etwa im mittleren frontalen Gyrus, im Bereich des inferioren frontalen Verbindungsareals, in den frontalen Augenfeldern und auch im intraparietalen Kortex. Darüber hinaus zeigte sich eine verminderte Aktivierung bei Patienten (Patienten < Kontrollen) unter anderem im rechten avPFC, linken anterioren mittleren frontalen Gyrus und im rechten parahippocampalen Gyrus. 
Tabelle 10: Differentielle Aktivierungen zwischen Patienten und Kontrollen im Aufgabenblock „Bonusverfolgung“ (D esire-Kontext).

\begin{tabular}{|c|c|c|c|c|}
\hline \multirow{2}{*}{ Himregion } & \multicolumn{2}{|c|}{ Patienten > Kontrollen } & \multicolumn{2}{|c|}{ Patienten < Kontrollen } \\
\hline & $\begin{array}{l}\text { Koordinate } \\
(\mathrm{x}, \mathrm{y}, \mathrm{z} ; \mathrm{mm})\end{array}$ & t-Wert & $\begin{array}{l}\text { Koordinate } \\
(\mathrm{x}, \mathrm{y}, \mathrm{z} ; \mathrm{mm})\end{array}$ & t-Wert \\
\hline L dorsales/ ventrales Striatum & $-1524-9$ & 3,4 & - & - \\
\hline $\begin{array}{l}\mathrm{R} \text { antero-ventraler präfrontaler } \\
\text { Kortex }\end{array}$ & - & - & 36639 & 2,8 \\
\hline $\begin{array}{l}\text { L anteriorer mittlerer frontaler } \\
\text { Gyrus }\end{array}$ & - & - & -395418 & 2,5 \\
\hline L mittlerer frontaler Gyrus & -333630 & 3,4 & - & - \\
\hline R mittlerer frontaler Gyrus & 364233 & 2,6 & - & - \\
\hline $\mathrm{R}$ inferiorer frontaler Sulkus & 333615 & 2,2 & - & - \\
\hline $\begin{array}{l}\text { L inferiores frontales } \\
\text { Verbindungsareal/ posteriorer } \\
\text { inferiorer frontaler Gyrus }\end{array}$ & -331533 & 3,8 & - & - \\
\hline $\begin{array}{l}\text { R inferiores frontales } \\
\text { Verbindungsareal/ posteriorer } \\
\text { inferiorer frontaler Gyrus }\end{array}$ & $42-648$ & 2,6 & - & - \\
\hline L frontales Augenfeld & $-36-1863$ & 3,1 & - & - \\
\hline $\mathrm{R}$ frontales Augenfeld & $27-351$ & 2,5 & - & - \\
\hline R mittlerer frontaler Gyrus & - & - & 39645 & 2,1 \\
\hline $\begin{array}{l}\text { L frontooperkularer Kortex/ } \\
\text { anteriore Insula }\end{array}$ & $-3024-12$ & 2,0 & - & - \\
\hline L intraparietaler Kortex & $-33-5727$ & 2,2 & - & - \\
\hline $\mathrm{R}$ intraparietaler Kortex & $33-7236$ & 1,8 & - & - \\
\hline
\end{tabular}




\begin{tabular}{|l|l|l|l|l|}
\hline L intraparietaler Kortex & $-36-4251$ & 2,3 & - & - \\
\hline L inferiorer parietaler Lobus & $-51-2142$ & 2,9 & - & - \\
\hline R inferiorer parietaler Lobus & $54-2739$ & 2,5 & - & - \\
\hline $\begin{array}{l}\text { L posteriorer frontomedianer } \\
\text { Kortex }\end{array}$ & - & - & -12654 & 1,9 \\
\hline $\begin{array}{l}\text { R posteriorer frontomedianer } \\
\text { Kortex }\end{array}$ & 15645 & 2,9 & - & - \\
\hline $\begin{array}{l}\text { L dorsaler anteriorer cingulärer } \\
\text { Kortex }\end{array}$ & -121530 & 2,1 & - & - \\
\hline R mittlerer temporaler Gyrus & - & - & $39-24-21$ & 2,5 \\
\hline R Cerebellum & - & - & $18-39-30$ & 2,0 \\
\hline $\begin{array}{l}\text { R Hippocampus/ } \\
\text { parahippocampaler Gyrus }\end{array}$ & - & - & $39-24-21$ & 2,4 \\
\hline
\end{tabular}

L: links; R: rechts
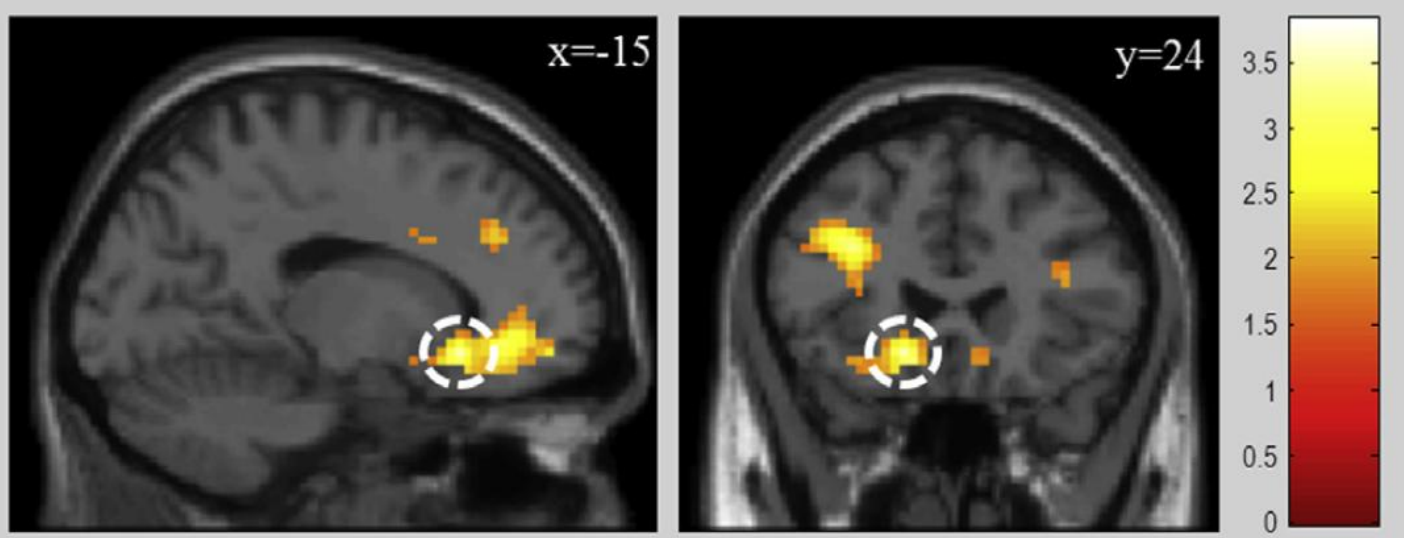

Abbildung 4: Gesteigerte Aktivierung im Aufgabenblock „Bonusverfolgung“ (D esireKontext) bei Patienten im Vergleich zu Kontrollpersonen (G ruppenvergleich Patienten > Kontrollen). Der Bereich des linken ventralen Striatums ist mit einem weißen Kreis markiert. Modifiziert aus Richter et al. (2015). 


\subsubsection{Reason-Kontext}

In diesem Teil der experimentellen Aufgabe mussten die positiv konditionierten Stimuli (Bonusreize) abgelehnt werden, um das übergeordnete Ziel innerhalb des Aufgabenblocks zu erreichen. Somit liegt eine Dilemmasituation vor, welche, wie in vorherigen Studien gezeigt werden konnte, mit einer signifikanten Aktivierung frontaler Areale und einer Unterdrückung der Aktivierung von belohnungs-assoziierten mesolimbischen Strukturen einhergeht (Diekhof und Gruber 2010; Diekhof et al. 2012a; Diekhof et al. 2012b). Entsprechend zeigten sich bei gesunden Kontrollpersonen Aktivierungen in frontalen Arealen, unter anderem im avPFC und auch im anterioren mittleren frontalen Gyrus, welche für die top-down-Regulation der Belohnungszentren maßgeblich sein könnten. Passend zu den oben erwähnten vorherigen Studien findet sich keine Aktivität im vStr in dieser Dilemmasituation. Abweichend vom Aktivierungsmuster der gesunden Kontrollpersonen sind bei Patienten die frontalen Aktivierungen im Bereich des avPFC und des anterioren mittleren frontalen Gyrus weniger stark ausgeprägt und es finden sich Aktivierungen im Bereich der bilateralen vStr (Tabelle 11). 
Tabelle 11: Aktivierungen der Patienten und Kontrollen im Aufgabenblock „Zielverfolgung“ (Reason-Kontext).

\begin{tabular}{|c|c|c|c|c|}
\hline \multirow{2}{*}{ H imregion } & \multicolumn{2}{|l|}{ Patienten } & \multicolumn{2}{|l|}{ Kontrollen } \\
\hline & $\begin{array}{l}\text { Koordinate } \\
(\mathrm{x}, \mathrm{y}, \mathrm{z} ; \mathrm{mm})\end{array}$ & t-Wert & $\begin{array}{l}\text { Koordinate } \\
(\mathrm{x}, \mathrm{y}, \mathrm{z} ; \mathrm{mm})\end{array}$ & t-Wert \\
\hline L dorsales/ ventrales Striatum & -1260 & 3,3 & - & - \\
\hline R dorsales/ ventrales Striatum & 1203 & 4,3 & $18-69$ & 5,2 \\
\hline L ventrale tegmentale Area & $-9-18-3$ & 3,3 & $-3-21-21$ & 2,0 \\
\hline $\mathrm{R}$ ventrale tegmentale Area & $6-27-18$ & 3,5 & $6-27-21$ & 1,8 \\
\hline $\begin{array}{l}\text { L antero-ventraler präfrontaler } \\
\text { Kortex }\end{array}$ & -304212 & 2,7 & -304512 & 2,8 \\
\hline $\begin{array}{l}\mathrm{R} \text { antero-ventraler präfrontaler } \\
\text { Kortex }\end{array}$ & - & - & 304812 & 3,4 \\
\hline $\begin{array}{l}\mathrm{L} \text { anteriorer mittlerer frontaler } \\
\text { Gyrus }\end{array}$ & -365121 & 2,1 & -365121 & 3,7 \\
\hline L mittlerer frontaler Gyrus & -333324 & 5,4 & - & - \\
\hline $\mathrm{R}$ mittlerer frontaler Gyrus & 393333 & 4,1 & 363030 & 4,1 \\
\hline $\mathrm{R}$ inferiorer frontaler Sulkus & 334221 & 5,4 & 334521 & 3,5 \\
\hline $\begin{array}{l}\text { L inferiores frontales } \\
\text { Verbindungsareal/ posteriorer } \\
\text { inferiorer frontaler Gyrus }\end{array}$ & -42033 & 5,5 & -45333 & 2,6 \\
\hline $\begin{array}{l}\mathrm{R} \text { inferiores frontales } \\
\text { Verbindungsareal/ posteriorer } \\
\text { inferiorer frontaler Gyrus }\end{array}$ & 48330 & 4,4 & 48333 & 3,8 \\
\hline $\begin{array}{l}\mathrm{R} \text { inferiores frontales } \\
\text { Verbindungsareal/ posteriorer } \\
\text { inferiorer frontaler Gyrus }\end{array}$ & - & - & 33033 & 4,5 \\
\hline
\end{tabular}




\begin{tabular}{|c|c|c|c|c|}
\hline L frontales Augenfeld & $-36-1863$ & 8,4 & $-36-1263$ & 5,1 \\
\hline $\mathrm{R}$ frontales Augenfeld & $24-948$ & 5,8 & $24-951$ & 4,3 \\
\hline $\begin{array}{l}\text { L frontooperkularer Kortex/ } \\
\text { anteriore Insula }\end{array}$ & -30243 & 5,0 & -30216 & 4,6 \\
\hline L posteriore Insula & $-39-39$ & 3,5 & $-39-39$ & 1,8 \\
\hline $\begin{array}{l}\text { R frontooperkularer Kortex/ } \\
\text { anteriore Insula }\end{array}$ & 33213 & 6,8 & 36213 & 6,0 \\
\hline $\mathrm{R}$ intraparietaler Kortex & $33-5442$ & 5,6 & $30-5142$ & 4,1 \\
\hline L intraparietaler Kortex & $-36-4548$ & 7,3 & $-27-5151$ & 4,7 \\
\hline L inferiorer parietaler Lobus & $-51-2745$ & 7,2 & $-45-3063$ & 6,3 \\
\hline $\mathrm{R}$ inferiorer parietaler Lobus & $45-3039$ & 6,3 & $42-3045$ & 5,7 \\
\hline R postzentraler Gyrus & - & - & $33-3048$ & 3,4 \\
\hline $\begin{array}{l}\text { L posteriorer frontomedianer } \\
\text { Kortex }\end{array}$ & -61245 & 7,9 & -61248 & 8,2 \\
\hline $\begin{array}{l}\text { L dorsaler anteriorer cingulärer } \\
\text { Kortex }\end{array}$ & -123330 & 1,8 & -182418 & 1,7 \\
\hline $\mathrm{R}$ cingulärer $\mathrm{G}$ yrus & - & - & $3-2724$ & 1,8 \\
\hline $\mathrm{R}$ mittlerer temporaler Gyrus & $39-453$ & 3,4 & $42-45-3$ & 3,7 \\
\hline L okzipitaler Kortex & $-15-96-9$ & 8,2 & $-18-969$ & 5,7 \\
\hline R okzipitaler Kortex & $21-999$ & 7,7 & $24-9612$ & 5,9 \\
\hline L Cerebellum & $-30-60-24$ & 5,7 & $-33-72-21$ & 5,7 \\
\hline L Thalamus & $-12-186$ & 3,2 & - & - \\
\hline
\end{tabular}

L: links; R: rechts 
D er Gruppenvergleich (Tabelle 12) zeigt eine verminderte Supression, also eine stärkere Aktivierung, in der bilateralen VTA und im bilateralen vStr bei Patienten (Patienten > Kontrollen; Abbildung 5). D arüber hinaus zeigt sich bei Patienten eine stärkere Aktivierung im frontoparietalen Kortex, unter anderem im linken mittleren frontalen Gyrus, im linken inferioren frontalen Verbindungsareal und im linken intraparietalen Kortex. Weiterhin zeigen Patienten eine Hypoaktivierung (Patienten < Kontrollen) im Bereich des avPFC, im Einklang mit einer verminderten top-down-Regulation und daraus resultierender relativen Mehraktivierung im bilateralen vStr und der bilateralen VTA.

Tabelle 12: Differentielle Aktivierungen zwischen Patienten und Kontrollen im Aufgabenblock „Zielverfolgung“ (Reason-Kontext).

\begin{tabular}{|c|c|c|c|c|}
\hline \multirow[b]{2}{*}{ H imregion } & \multicolumn{2}{|c|}{ Patienten > Kontrollen } & \multicolumn{2}{|c|}{ Patienten < Kontrollen } \\
\hline & $\begin{array}{l}\text { Koordinate } \\
(\mathrm{x}, \mathrm{y}, \mathrm{z} ; \mathrm{mm})\end{array}$ & $\mathrm{t}$-Wert & $\begin{array}{l}\text { Koordinate } \\
(\mathrm{x}, \mathrm{y}, \mathrm{z} ; \mathrm{mm})\end{array}$ & t-Wert \\
\hline L dorsales/ ventrales Striatum & $-1212-12$ & 3,1 & - & - \\
\hline $\mathrm{R}$ dorsales/ ventrales Striatum & $1512-15$ & 2,5 & 9318 & 2,9 \\
\hline L ventrale tegmentale Area & $-15-15-12$ & 2,2 & - & - \\
\hline $\mathrm{R}$ ventrale tegmentale Area & $3-30-9$ & 1,9 & - & - \\
\hline $\begin{array}{l}\mathrm{R} \text { antero-ventraler präfrontaler } \\
\text { Kortex }\end{array}$ & - & - & 36639 & 2,3 \\
\hline $\begin{array}{l}\text { L anteriorer mittlerer frontaler } \\
\text { G yrus }\end{array}$ & - & - & -395418 & 1,8 \\
\hline L mittlerer frontaler Gyrus & -332727 & 3,7 & - & - \\
\hline R inferiorer frontaler Sulkus & 33361 & 2,7 & - & - \\
\hline $\begin{array}{l}\text { L inferiores frontales } \\
\text { Verbindungsareal/ posteriorer } \\
\text { inferiorer frontaler Gyrus }\end{array}$ & $-51-336$ & 2,7 & - & - \\
\hline
\end{tabular}




\begin{tabular}{|c|c|c|c|c|}
\hline $\begin{array}{l}\mathrm{R} \text { inferiores frontales } \\
\text { Verbindungsareal/ posteriorer } \\
\text { inferiorer frontaler Gyrus }\end{array}$ & $42-627$ & 1,8 & - & - \\
\hline $\begin{array}{l}\mathrm{R} \text { inferiores frontales } \\
\text { Verbindungsareal/ posteriorer } \\
\text { inferiorer frontaler Gyrus }\end{array}$ & - & - & 36339 & 3,0 \\
\hline L frontales Augenfeld & $-36-1560$ & 2,4 & - & - \\
\hline $\mathrm{R}$ frontales Augenfeld & $24-1245$ & 1,8 & - & - \\
\hline L posteriore Insula & $-45-123$ & 2,6 & - & - \\
\hline $\begin{array}{l}\text { R frontooperkularer Kortex/ } \\
\text { anteriore Insula }\end{array}$ & 362718 & 2,1 & - & - \\
\hline $\mathrm{R}$ intraparietaler Kortex & $27-7239$ & 2,5 & - & - \\
\hline L intraparietaler Kortex & $-36-4545$ & 4,0 & - & - \\
\hline L inferiorer parietaler Lobus & $-51-2745$ & 2,7 & - & - \\
\hline $\mathrm{R}$ inferiorer parietaler Lobus & $54-2727$ & 3,3 & - & - \\
\hline R postzentraler Gyrus & - & - & $33-3048$ & 2,6 \\
\hline $\begin{array}{l}\text { L posteriorer frontomedianer } \\
\text { Kortex }\end{array}$ & -182154 & 3,1 & - & - \\
\hline $\begin{array}{l}\text { L dorsaler anteriorer cingulärer } \\
\text { Kortex }\end{array}$ & -123330 & 2,5 & - & - \\
\hline $\mathrm{R}$ cingulärer $\mathrm{G}$ yrus & - & - & $0-3012$ & 1,9 \\
\hline $\mathrm{R}$ mittlerer temporaler Gyrus & - & - & $33-42-27$ & 1,8 \\
\hline L Cerebellum & $-30-63-15$ & 1,9 & - & - \\
\hline L Thalamus & $-18-240$ & 2,1 & - & - \\
\hline
\end{tabular}

L: links; R: rechts 

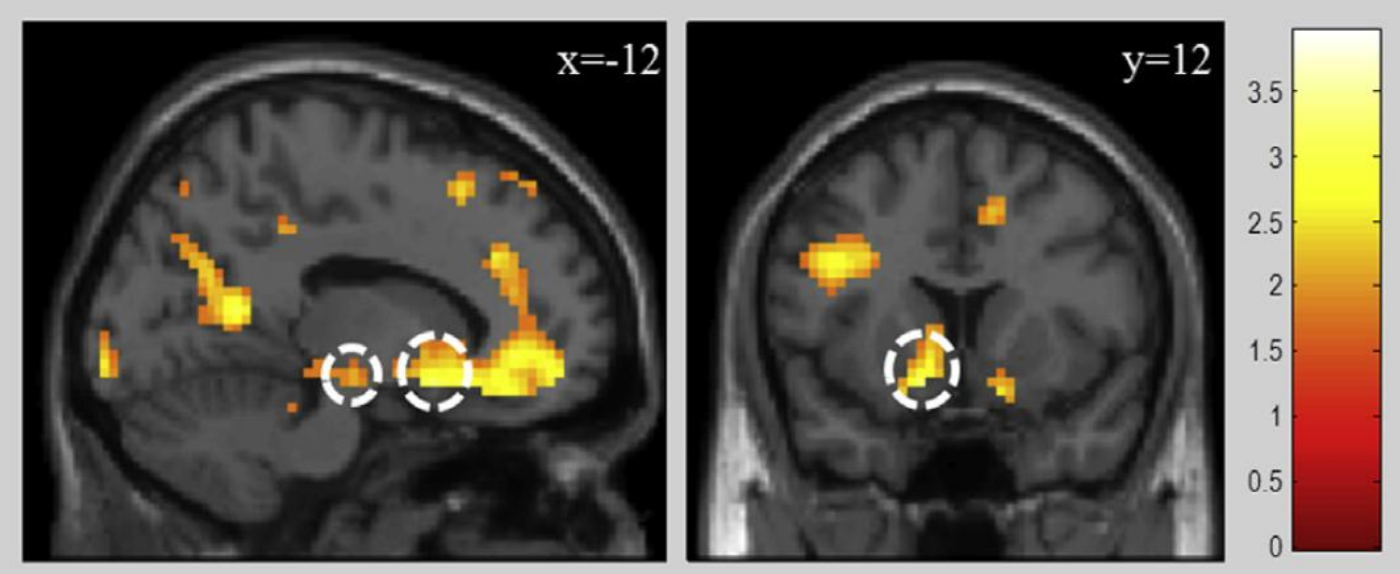

Abbildung 5: Gesteigerte Aktivierung im Aufgabenblock „Zielverfolgung“ (ReasonKontext) bei Patienten im Vergleich zu Kontrollpersonen (G ruppenvergleich Patienten > Kontrollen). Die Bereiche des linken ventralen Striatums und der ventralen tegmentalen Area sind mit weißen Kreisen markiert. Modifiziert aus Richter et al. (2015).

\subsubsection{Psychophysiologische Interaktion (PPI)}

Für die PPI-Analyse wurden als Startregionen (regions-of-interest) bilaterale Regionen im Bereich des vStr gewählt. Im Einklang mit vorherigen Ergebnissen aus unserer Arbeitsgruppe (D iekhof und Gruber 2010) zeigte sich bei gesunden Kontrollpersonen in der Dilemmasituation eine signifikant erhöhte negative funktionelle Interaktion zwischen rechtem vStr und bilateralem avPFC sowie linkem vStr und bilateralem avPFC. D arüber hinaus zeigte sich in dieser Gruppe eine ebenfalls signifikant erhöhte negative funktionelle Interaktion zwischen rechtem bzw. linkem vStr und ventralem medialem präfrontalem Kortex (VMPFC) (Tabelle 13).

Im Vergleich zu gesunden Kontrollpersonen zeigte sich bei Patienten eine signifikant reduzierte negative Interaktion zwischen vStr und avPFC sowie zwischen vStr und VMPFC (Tabelle 14, Abbildung 6). 
Tabelle 13: Gesteigerte negative funktionelle Interaktion bei Kontrollen in der D ilemmasituation, ausgehend von Regionen im ventralen Striatum.

\begin{tabular}{|l|l|l|}
\hline & \multicolumn{2}{|l|}{ Kontrollen } \\
\hline H imregion & $\begin{array}{l}\text { Koordinate } \\
(\mathrm{x}, \mathrm{y}, \mathrm{z} ; \mathrm{mm})\end{array}$ & t-Wert \\
\hline Startregion: L ventrales Striatum & & \\
\hline L antero-ventraler präfrontaler Kortex & -12483 & 3,3 \\
\hline L ventraler medialer präfrontaler Kortex & $-939-6$ & 3,5 \\
\hline R ventraler medialer präfrontaler Kortex & $642-12$ & 4,4 \\
\hline Startregion: R ventrales Striatum & & \\
\hline L antero-ventraler präfrontaler Kortex & -12483 & 3,5 \\
\hline R antero-ventraler präfrontaler Kortex & $1251-3$ & 3,6 \\
\hline L ventraler medialer präfrontaler Kortex & $-639-9$ & 3,5 \\
\hline R ventraler medialer präfrontaler Kortex & $642-9$ & 4,8 \\
\hline
\end{tabular}

L: links; R: rechts 
Tabelle 14: Reduzierte negative funktionelle Interaktion bei Patienten im Vergleich zu Kontrollen in der Dilemmasituation, ausgehend von Regionen im ventralen Striatum.

\begin{tabular}{|l|l|l|}
\hline & \multicolumn{2}{|l|}{ Patienten } \\
\hline H imregion & $\begin{array}{l}\text { Koordinate } \\
(\mathrm{x}, \mathrm{y}, \mathrm{z} ; \mathrm{mm})\end{array}$ & t-Wert \\
\hline Startregion: L ventrales Striatum & & 1,9 \\
\hline L antero-ventraler präfrontaler Kortex & -12519 & 3,5 \\
\hline R antero-ventraler präfrontaler Kortex & 12399 & 2,0 \\
\hline L ventraler medialer präfrontaler Kortex & $-1242-9$ & 2,5 \\
\hline R ventraler medialer präfrontaler Kortex & $642-12$ & \\
\hline Startregion: R ventrales Striatum & & 2,7 \\
\hline L antero-ventraler präfrontaler Kortex & -15453 & 3,2 \\
\hline R antero-ventraler präfrontaler Kortex & $1251-3$ & 2,3 \\
\hline L ventraler medialer präfrontaler Kortex & $-1242-9$ & 3,4 \\
\hline R ventraler medialer präfrontaler Kortex & $939-6$ & \\
\hline
\end{tabular}

L: links; R: rechts 


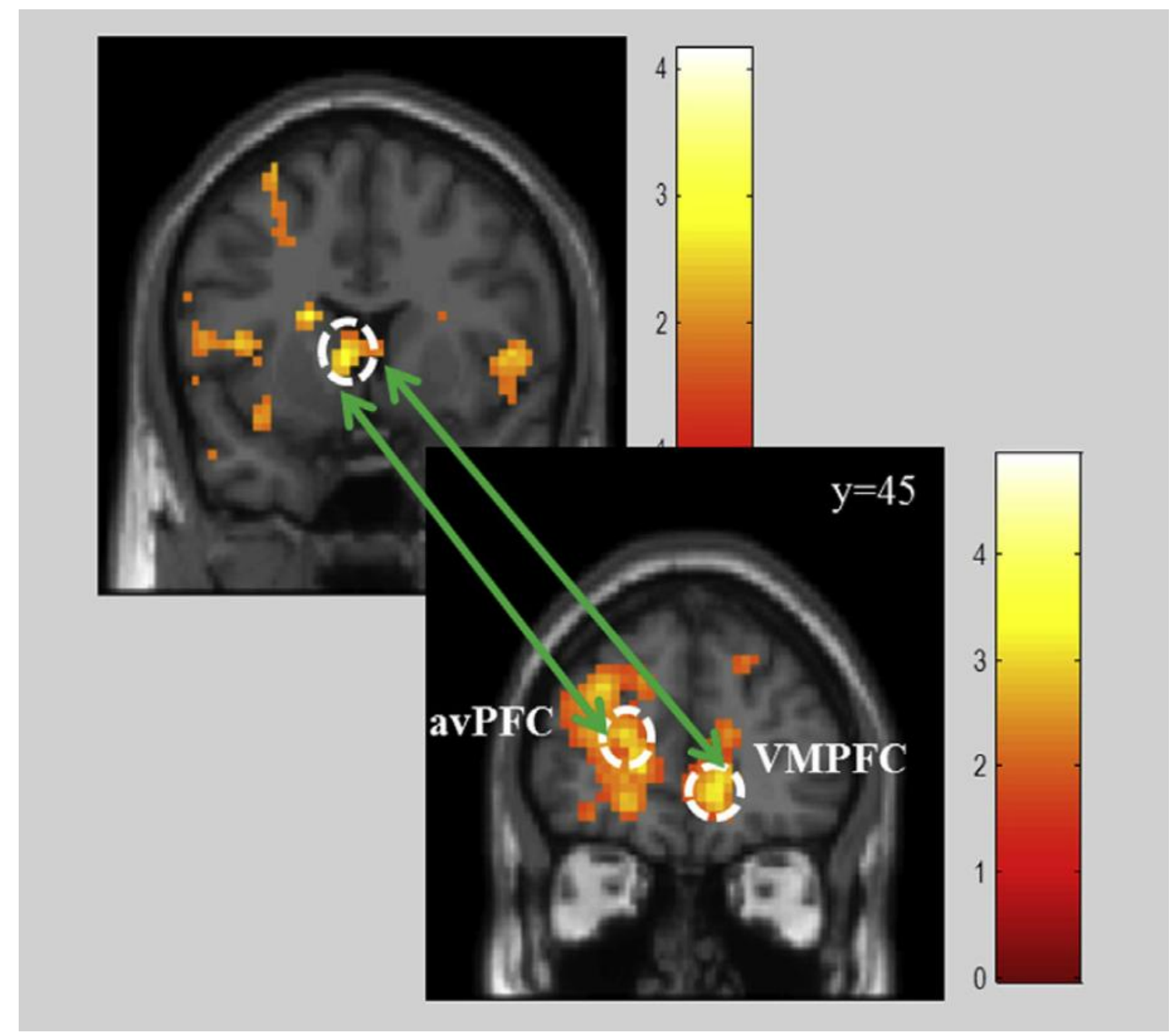

Abbildung 6: Die PPI-Analyse zeigt eine reduzierte negative funktionelle Interaktion zwischen linkem ventralem Striatum (oben links) und dem antero-ventralen präfrontalen Kortex (avPFC) sowie dem ventralen medialen präfrontalen Kortex (VMPFC) bei Patienten im Vergleich zu Kontrollen in der Dilemmasituation. Modifiziert aus Richter et al. (2015) . 


\section{Diskussion}

In der vorliegenden Arbeit untersuchte ich unter Einsatz der fMRT und eines Verhaltensparadigmas, welches in der kritischen Phase des Experimentes bei der Versuchsperson die Unterdrückung von Belohnungsreizen erfordert, um ein langfristiges Ziel zu erreichen (Dilemmasituation), inwieweit sich Patienten mit Schizophrenie von gesunden Kontrollpersonen hinsichtlich der erforderlichen Impulskontrolle und auch der Aktivierbarkeit des Belohnungssystems unterscheiden. Es wurden jeweils 16 Patienten und 16 Kontrollpersonen untersucht.

In der Bonusverfolgung, in welcher die vorher konditionierten Reize angenommen werden konnten, zeigten die Patienten im Vergleich zu Kontrollpersonen als Kernergebnis eine gesteigerte Aktivität im vStr, im Sinne einer bottom-up-Aktivierung durch den Bonusreiz, und eine verminderte Aktivität in frontalen Arealen. In der Zielverfolgung des Experimentes (Dilemmasituation) zeigten sich zum einen die erwarteten Effekte auf Verhaltensebene, nämlich ein Defizit in der Ablehnung von Belohnungsreizen. Zum anderen konnten auf neurofunktioneller Ebene Korrelate dieser Defizite identifiziert werden. Es zeigte sich bei Patienten im Vergleich zu Kontrollpersonen eine Hyperaktivierung des vStr und der VTA, also eine verstärkte bottom-up-Aktivierung, bei gleichzeitiger Hypoaktivierung frontaler Areale im Einklang mit einer verminderten topdown-Impulskontrolle. Zudem konnte eine verminderte funktionelle Konnektivität im Sinne einer gestörten top-down-Modulation zwischen frontalen Arealen und dem vStr gezeigt werden.

\subsection{Mesolimbisches System - bottom-up-Regulation}

Unter Einsatz des verwendeten Paradigmas zeigten Patienten mit Schizophrenie eine erhöhte A ktivität im mesolimbischen System. Insbesondere die Überaktivität im vStr passt zur Theorie des Ungleichgewichts des dopaminergen Systems bei Schizophrenen. Störungen im Dopamingleichgewicht im Sinne einer vermehrten Dopaminfreisetzung bei gleichzeitig mangelnder D 2-Rezeptorbindung konnten bereits in früheren Studien gezeigt werden (A bi-D argham et al. 1998; A bi-D argham et al. 2000).

Ein gängiges Modell geht davon aus, dass bei Schizophrenen - aufgrund einer fehlregulierten D opaminübetragung - an sich irrelevante Stimuli als wichtig erachtet werden (Kapur 2003). Im Einklang mit diesem Modell zeigte sich in einer Studie mit 
Schizophreniepatienten, in der ein Pavlov'sches Konditionierungsparadigma verwendet wurde, eine Hyperaktivierung im vStr (Romaniuk et al. 2010). In einer anderen Studie, welche Individuen mit einem hohen Risiko für die Ausbildung einer Psychose mit einer Aufgabe konfrontierte, die relevante und irrelevante Stimuli enthielt, konnte ebenfalls eine abnormale Aktivität im vStr gezeigt werden (Roiser et al. 2013).

D as vStr (insbesondere der Nacc) spielt eine zentrale Rolle im Belohnungssystem, da es zahlreiche Verbindungen mit erregenden, hemmenden oder modulierenden Einflüssen vom Kortex und mesolombischen System vereinen muss. Diese Integration der verschiedenen Einflüsse wird durch in der VTA freigesetztes Dopamin gesteuert (Haber und Knutson 2010). Andererseits hat auch der Nacc Einfluss auf den D opaminhaushalt der VTA (Sesack und Grace 2009). Es liegt also eine Rückkopplungsschleife zwischen VTA und Nacc vor. In der vorliegenden Arbeit zeigte sich eine gesteigerte Aktivität im vStr, wenn konditionierte Reize angenommen werden durften.

Die hier gezeigte Überaktivierung des vStr bei Patienten mit Schizophrenie scheint nicht mit den Ergebnissen einiger vorheriger Studien im Einklang zu stehen. So zeigte sich beispielsweise in Experimenten bezüglich Belohnungserwartung eine abgeschwächte Antwort des vStr (Juckel et al. 2006a; G rimm et al. 2014). Jedoch ist zu beachten, dass in diesen Studien verschiedene Varianten der IncentiveM onetary-D elay-A ufgabe verwendet wurden. Bei dieser Kategorie von Aufgaben müssen die Versuchspersonen, nachdem sie einen Hinweisreiz mit einer Information hinsichtlich des zu erwartenden Gewinns bzw. Verlustes bekommen haben, auf einen folgenden Reiz reagieren. Die Rückmeldung über den entsprechenden Gewinn oder Verlust erfolgt hierbei sofort im Anschluss an die Antwort der Versuchsperson. Somit sind für diese Kategorie von Verhaltensexperimenten intakte Prozesse der Erwartungshaltung und Motivation zwingend notwendig. Dies gilt nicht für das hier verwendete Verhaltensparadigma, das Desire-Reason-D ilemma (DRD)Paradigma, in dem Stimuli konditioniert und präsentiert werden, welche mit einer Belohnung einhergehen. Dadurch war es möglich, den direkten Einfluss der Belohnungsreize ohne zusätzliche Prozesse der Erwartungshaltung zu untersuchen. Es konnte somit die sofortige bottom-up-Antwort des dopaminergen Belohnungssystems auf den konditionierten Stimulus erfasst werden. Folglich lassen sich diese scheinbar gegensätzlichen Ergebnisse wie folgt interpretieren: Patienten mit Schizophrenie zeigen eine Überaktivierung des Belohnungssystems aufgrund von direkter Stimulation mit Belohnungsreizen - im Einklang mit der Theorie eines Ungleichgewichts des dopaminergen Systems und entsprechenden Studienergebnissen (Abi-D argham et al. 1998; Abi-D argham 
et al. 2000; Kapur 2003; Romaniuk et al. 2010; Roiser et al. 2013), wohingegen bei der IncentiveM onetary-D elay-Aufgabe gestörte Erwartungsprozesse zu einer Unteraktivierung des Belohnungssystems führen. In entsprechender Weise lassen sich auch die Ergebnisse einer Studie einordnen, in welcher ein Pavlov'sches Belohnungsvorhersage-Paradigma eingesetzt wurde (Dowd und Barch 2012). Bei dieser Aufgabe wird zwar ein Belohnungsreiz konditioniert, im Verlauf des Paradigmas wird aber auf eine Antwort der Versuchsperson verzichtet. Es zeigten sich weitgehend normale neuronale Antworten auf Belohnungsreize bei chronisch Schizophrenen. Bei den Patienten mit stärker ausgeprägter Symptomatik zeigte sich jedoch eine abgeschwächte Antwort des vStr in der Phase der Belohnungserwartung, was wie bei der Inœentive M onetary-D elay-Aufgabe auf den Einfluss der gestörten Erwartungsprozesse auf das Belohnungssystem hinweist.

Weiter zeigte sich anhand der Performanzdaten bei Patienten mit Schizophrenie eine signifikant erniedrigte Rate an korrekt angenommenen Stimuli in der Bonusverfolgung. Dies könnte eine verminderte bottom-up-Aktivierung des Belohungssystems in der Patientengruppe erwarten lassen. Tatsächlich geht dieses Performanzdefizit allerdings mit einer gesteigerten Aktivität des vStr bei den Patienten einher.

In gesunden Versuchspersonen zeigte sich bei Ablehnung eines zuvor konditionierten Stimulus im Aufgabenblock „Zielverfolgung“ eine signifikant abgeschwächte Antwort im mesolimbischen Belohnungssystem. Darüber hinaus konnte eine signifikante, negative Kopplung zwischen präfrontalen Regionen und dem vStr gezeigt werden. Diese Ergebnisse stimmen sehr gut mit Resultaten aus vorherigen Studien unserer Arbeitsgruppe überein (Diekhof und Gruber 2010; D iekhof et al. 2012a; Diekhof et al. 2012b) und deuten auf die Reliabilität des verwendeten Verhaltensparadigmas hin. Im Gegensatz zu den Effekten bei gesunden Kontrollpersonen zeigten Patienten mit Schizophrenie eine signifikant verminderte Unterdrückung der Belohnungssignale in Bereichen des bilateralen vStr und der bilateralen VTA. Zusätzlich fanden sich gesteigerte Aktivierungen in weitreichenden frontalen und parietalen Kortexarealen.

D as Kernergebnis der vorliegenden Arbeit ist sicherlich das Aufdecken einer signifikant beeinträchtigten top-down-Regulation des mesolimbischen Systems durch den präfrontalen Kortex in der Dilemmasituation. D ieses Ergebnis steht im Einklang mit dem Modell einer veränderten Konnektivität bei Schizophrenie (Stephan et al. 2006). Passend hierzu zeigte sich in einer kürzlich durchgeführten fMRT-Studie mit einer Arbeitsgedächtnisaufgabe bei Patienten mit Schizophrenie eine gestörte Kopplung zwischen dem präfrontalen Kortex 
und der Substantia nigra (Yoon et al. 2013). Weiter konnte in einer Positronenemissionstomografie-Studie gezeigt werden, dass Patienten mit Schizophrenie während einer Arbeitsgedächtnisaufgabe eine Minderaktivierung in präfrontalen Regionen aufweisen. Gleichzeitig war die Aufnahmekonstante für Dopamin im Striatum erhöht, sodass die Interpretation naheliegt, dass es einen Zusammenhang zwischen einer gestörten Funktion des präfrontalen Kortex und einer abnormalen Dopaminübertragung geben könnte (Meyer-Lindenberg et al. 2002).

\subsection{Frontalkortex - top-down-Regulation}

Neben den oben beschriebenen Veränderungen im mesolimbischen System zeigten sich bei der Patientengruppe weitere Auffälligkeiten in verschiedenen kortikalen Hirnregionen, welche mit Funktionen des Arbeitsgedächtnisses, mit Aufmerksamkeitprozessen und kognitiven Funktionen in Verbindung gebracht werden (Gruber und Goschke 2004). Im Folgenden soll auf die abweichende kortikale Hirnaktivierung in frontalen Arealen in der Patientengruppe eingegangen werden. So zeigte sich in verschiedenen präfrontalen Regionen eine Hyper- bzw. Hypoaktivierung. D iese könnten zumindest teilweise auf die gesteigerten Anforderungen an das Arbeitsgedächtnis in der Patientengruppe im Vergleich zur Kontrollgruppe zurückzuführen sein. In einem bekanntem Model (Manoach 2003) wird die präfrontale Hyperaktivierung bei Patienten mit Schizophrenie als eine mögliche Folge einer reduzierten Kapazität des Arbeitsgedächtnisses gesehen. Kommt es nun aber zu sehr hohen Anforderungen an das Arbeitsgedächtnis, welche die Kapazität des Arbeitsgedächtnisses der Patienten überschreiten, könnte daraus eine präfrontale Hypoaktivierung resultieren.

D ie in der vorliegenden Arbeit gezeigten Ergebnisse einer präfrontalen Hyperaktivierung passen somit sehr gut zu früheren Arbeiten unter Verwendung der fMRT (Manoach et al. 1999; Manoach et al. 2000). D iese fanden unter Einsatz von Arbeitsgedächtnisaufgaben mit und ohne Belohnung bei Patienten mit Schizophrenie ebenfalls eine gesteigerte Aktivität in frontalen Arealen, nämlich im dorsolateralen präfrontalen Kortex.

Des Weiteren zeigte sich ebenfalls eine Hypoaktivierung in anterioren Teilen des Frontalkortex, insbesondere im avPFC und anterioren mittleren frontalen Gyrus. Dies ist im Einklang mit früheren Studien, welche ebenfalls eine vermindert Aktivierung in frontalen Arealen bei Arbeitsgedächtnisaufgaben fanden (Barch et al. 2001; Perlstein et al. 2001). Diese Ergebnisse weisen auf eine präfrontale Dysfunktion bei Schizophrenen hin, 
welche in einer gestörten Verbindung zwischen präfrontalem Kortex und mesolimbischem Belohnungssystem resultiert und somit im Sinne einer beeinträchtigten top-down-Regulation des Belohnungssystems durch den avPFC interpretiert werden kann.

Darüber hinaus zeigte die Patientengruppe in der vorliegenden Arbeit eine Hyperaktivierung im frontoopercularen Kortex. Dieser Hirnbereich ist Teil des Arbeitsgedächtnisses und beim Erhalt und Abruf von Gedächtnisinhalten involviert (Gruber und von Cramon 2003). Ebenso fand sich eine signifikant gesteigerte Aktivierung in den bilateralen frontalen Augenfeldern, einer Region, die in okulomotorische Prozesse, visuelle Aufmerksamkeit und in die Aufrechterhaltung von visuellen Informationen involviert ist (Gruber und von Cramon 2001; G ruber und von Cramon 2003). Führt man sich einmal mehr das hier verwendete Verhaltensparadigma vor Augen, so können die Aktivierungen in diesen Regionen gut eingeordnet werden. Um die Aufgabe erfolgreich zu bewältigen, mussten sich die Teilnehmer der Studie die beiden Zielfarben zu Beginn eines jeden Durchgangs merken. Die gesteigerten Aktivitäten in den genannten Regionen bei Patienten mit Schizophrenie könnten also die größere Anstrengung des Merkens und des A brufs der aufgabenrelevanten Informationen, die verschiedenen Zielfarben, reflektieren.

Hyperaktivierungen wurden im inferioren frontalen Verbindungsareal und im mittleren frontalen Kortex beobachtet. Diese Regionen spielen eine wichtige Rolle im Zusammenhang mit kognitiver Kontrolle (D errfuss et al. 2004; G ruber und Goschke 2004; Gruber et al. 2009). Wenn man einen Zusammenhang zwischen exekutiven Kontrollanforderungen und frontaler Aktivität in Betracht zieht (Bellgrove et al. 2004), so könnte diese Hyperaktivierung Ausdruck einer zusätzlichen Anstrengung sein, die bei den Patienten notwendig ist, um die Aufgabe zu bewältigen (Arce et al. 2006).

Insgesamt lässt sich also festhalten, dass wir bei unserem Patientenkollektiv ausgedehnte Hyperaktivitäten in frontalen und parietalen Netzwerken gefunden haben. D iese könnten durch eine fälschlich wahrgenommene gesteigerte Salienz der Belohnungsreize verursacht sein, was zu Aktivierungen in entsprechenden Netzwerken des Arbeitsgedächtnisses, der Aufmerksamkeitskontrolle und der kognitiven Kontrolle führt. Allerdings lässt sich aufgrund der erhobenen Daten nicht unterscheiden, ob die Ergebnisse tatsächlich die veränderte Salienz oder einfach nur die Ineffizienz der frontoparietalen Netzwerke im Zusammenhang mit der gestellten Verhaltensaufgabe in den Patienten mit Schizophrenie widerspiegeln. 


\subsection{Methodische Aspekte}

Wie jede wissenschaftliche Studie hat auch diese Arbeit einige Limitationen, welche bei der Interpretation der Ergebnisse berücksichtigt werden müssen.

So wurden trotz umfangreicher Rekrutierungsbemühungen am Ende nur 16 Patienten mit Schizophrenie in die Studie eingeschlossen. Die Rekrutierungsphase dauerte mehrere Monate. Allerdings sind gerade psychiatrische Patienten, insbesondere solche mit einer Schizophrenie, aufgrund ihrer Symptomatik ein sehr schwieriges Kollektiv. D ennoch liegt die Patientenzahl in der vorliegenden Arbeit mit 16 Teilnehmern am oberen Ende der Kollektivgröße im Vergleich mit anderen Studien, die sich ebenfalls mit Aspekten des Belohnungssystems beschäftigt haben. So schlossen beispielsweise Juckel et al. (2006a) nur 10 Patienten ein. Bei der Studie von Abler et al. (2007) waren es immerhin 12 und bei Schlagenhauf et al. (2009) 15 Patienten. Gerade weil die Aussagekraft bei kleinen Patientenkollektiven generell eingeschränkt ist, legten wir besonderen Wert auf ein gutes Matching der Kontrollgruppe. Nichtsdestotrotz sind weitere Studien mit größeren Patientenzahlen erstrebenswert.

Um die unterschiedlichen Hirnaktivitätsmuster zweifelsfrei dem Faktor „Krankheit" zuordnen zu können, müssten alle anderen Faktoren zwischen den beiden untersuchten $\mathrm{G}$ ruppen gleich gehalten werden. Aus diesem $\mathrm{G}$ rund wurden die beiden Gruppen sehr sorgfältig gematcht. Dennoch gibt es zwei Aspekte, die über den Krankheitsfaktor hinaus zwischen den G ruppen unterschiedlich sind.

Ein Aspekt, welcher aus ethischen Gründen nicht umgangen werden kann, sind die individuellen Medikationen der Patienten. Je nach Medikation ist ein Einfluss auf das dopaminerge System, und damit auf für die vorliegende Studie wichtige Hirnregionen, nicht auszuschließen. Allerdings wäre in diesem Fall eine Beeinträchtigung der dopaminergen Übertragungswege zu erwarten, was aufgrund der Regelschleife zwischen VTA und Nacc zu einer Hypoaktivierung des vStr führen sollte (Juckel et al. 2006b). Wir fanden bei den untersuchten Patienten allerdings eine Hyperaktivierung des vStr. Somit scheint ein direkter Einfluss der Medikation auf die Ergebnisse der vorliegenden Studie unwahrscheinlich. D ennoch sollte dies bei der Einordnung der Ergebnisse nicht gänzlich außer Acht gelassen werden.

Ein anderer Aspekt sind die unterschiedlichen Performanzleistungen zwischen den G ruppen. Die Patienten schnitten hier durchweg schlechter ab. Nun hängen aber diese verschlechterte Performanz auf der einen Seite und ein verändertes Hirnaktivitätsmuster 
auf der anderen Seite miteinander zusammen. Deshalb sollten die identifizierten D ysfunktionen der subkortikalen und kortikalen Netzwerke nicht nur auf die D iagnose der Schizophrenie alleine zurückgeführt werden, sondem immer auch unter Berücksichtigung der unterschiedlichen Performanzleistungen eingeordnet werden.

In der vorliegenden Arbeit wurde die fMRT zur Charakterisierung von Hirnaktivitätsänderungen eingesetzt. Wichtig ist in diesem Zusammenhang zu berücksichtigen, dass es sich bei der fMRT um ein indirektes Verfahren handelt. Die gemessenen Signaländerungen spiegeln nicht direkt Änderungen der neuronalen Aktivität wieder, sondern sind über veränderte Durchblutung und Oxygenierung des Hirngewebes vermittelt (D erntl et al. 2010; Huettel et al. 2014).

\subsection{Ausblick}

Die Ergebnisse der vorliegenden Arbeit könnten in verschiedenen Bereichen der (klinischen) Forschung bei Erkrankungen des schizophrenen Formenkreises als G rundlage für weiterführende Untersuchungen dienen.

So könnte bei Einsatz des D RD-Paradigmas anhand von Himaktivierungsmustern in der D ilemmasituation die D iagnosesicherung einer Schizophrenie unterstützt werden. Zudem könnte es möglich sein, die Erkrankungen des schizophrenen Formenkreises anhand dieser Hirnaktivierungsmuster in entsprechende bekannte Untergruppen einzuteilen oder sogar neue Subgruppen zu identifizieren. Hierfür sind weiterführende Studien mit einer größeren Anzahl an Patienten aus den verschiedenen Untergruppen der schizophrenen Erkrankungen notwendig. Die Rekrutierung geeigneter Patienten gestaltete sich schon in der vorliegenden Arbeit schwierig. Bei einer angestrebten größeren Fallzahl unter Berücksichtigung weiterer Subgruppen der Erkrankungen des schizophrenen Formenkreises dürfte sich die Rekrutierungssituation weiter verschärfen, sodass solche Untersuchungen in Multizenter-Studien durchgeführt werden sollten.

D as hier verwendete experimentelle Paradigma könnte auch bei gesunden Personen, die jedoch eine gewisse Prädisposition für eine schizophrene Erkrankung haben, eingesetzt werden. So könnten, bei einem Hinweis auf eine erhöhte Wahrscheinlichkeit für das Entwickeln einer Erkrankungen des schizophrenen Formenkreises, bereits frühzeitig vorbeugende Maßnahmen eingeleitet werden. Eine Studie aus unserer Arbeitsgruppe mit gesunden erstgradigen Angehörigen von Patienten mit einer schizophrenen Erkrankung konnte bereits zeigen, dass es auch bei den Angehörigen im Vergleich zu gesunden 
Kontrollpersonen zu einer gesteigerten Aktivität im mesolimbischen System in der D ilemmasituation kommt (Fanelli 2013).

Ein weiteres Einsatzgebiet könnte die Identifikation von dysfunktionalen Regionen für eine gezielte Therapie bei Patienten mit einer schizophrenen Erkrankung sein. Hier kommen sowohl pharmakologische Therapien als auch elektrophysiologische Therapieansätze infrage. So könnte beispielsweise mittels transkranieller Magnetstimulation eine gezielte Aktivierung von frontalen Hirnarealen versucht werden, um die fehlende top-downSuppression der mesolimbischen Aktivierungen bei den Patienten wieder herzustellen. Anhand von therapiebegleitenden fMRT-Untersuchungen könnten die erzielten Behandlungseffekte anhand der Hirnaktivitätsmuster verfolgt und evaluiert werden. 


\section{Zusammenfassung}

D ie Schizophrenie ist ein häufiges psychiatrisches Erkrankungsbild, mit stark heterogener Klinik und Ausprägung. Aufgrund der komplexen Manifestation der Schizophrenie gestaltet sich eine Diagnosestellung oftmals sehr schwierig, und es scheint auch innerhalb einer als schizophren diagnostizierten Gruppe Untergruppen zu geben. Durch Untersuchungen mit himbildgebenden Verfahren konnten Funktionen und Dysfunktionen neuronaler Systeme identifiziert werden. Ein neuronales System, welches im Zusammenhang mit der Schizophrenie von besonderer Bedeutung zu sein scheint, ist das dopaminerge Belohnungssystem bzw. das mesolimbische System.

In der vorliegenden Arbeit wurde mittels fMRT und einem etablierten Verhaltensparadigma das dopaminerge Belohnungssystem bei an Schizophrenie erkrankten Patienten im Vergleich zu gesunden Kontrollpersonen untersucht. Es kam das DRDParadigma (Diekhof und Gruber 2010) zum Einsatz, welches die Identifizierung von Arealen erlaubt, die in Entscheidungsprozesse und Belohnungsverarbeitung involviert sind. In diesem DRD-Paradigma wird eine Dilemmasituation herbeigeführt, in der die Versuchsperson vorher konditionierte Belohnungsreize ablehnen muss, um ein übergeordnetes Ziel zu erreichen. Dadurch lässt sich sowohl die Aktivierbarkeit des Belohnungssystems als auch die Impulskontrolle untersuchen.

Durch den Vergleich zwischen an Schizophrenie erkrankten Patienten und gesunden Kontrollpersonen konnten Aktivitätsunterschiede gefunden werden, welche möglicherweise neurofunktionelle Korrelate der Erkrankung darstellen. So zeigten die Patienten im Vergleich zu Kontrollen in der Dilemmasituation eine Hyperaktivierung des vStr und der VTA, also eine verstärkte bottom-up-Aktivierung des Belohnungssystems, bei gleichzeitiger Hypoaktivierung in bestimmten frontalen Arealen, im Einklang mit einer verminderten top-down-Impulskontrolle. Zudem konnte eine verminderte funktionelle Konnektivität im Sinne einer gestörten top-down-Modulation zwischen frontalen Arealen und dem vStr gezeigt werden.

Abschließend lässt sich festhalten, dass mit den vorliegenden Untersuchungen bei Patienten mit Schizophrenie Veränderungen auf neurofunktioneller Ebene identifiziert werden konnten. D as Wissen um die betroffenen Areale und deren D ysfunktion könnte in Zukunft die Möglichkeit bieten, dieses Wissen in die individuelle Therapie miteinzubeziehen und somit gezielter in die betroffenen Netzwerke einzugreifen. In Verbindung mit weiterführenden Untersuchungen an Subgruppen von Erkrankten des 
schizophrenen Formenkreises könnte die Charakterisierung der neuronalen Dysfunktion noch weiter verfeinert werden mit dem Ziel, die Symptome der Patienten zu lindern und deren subjektives Leidensgefühl zu schmälern. 


\section{Anhang}

\subsection{Messparameter für die Magnetresonanztomografie}

SIEMENS MAGNETOM TrioTim syngo MR B17

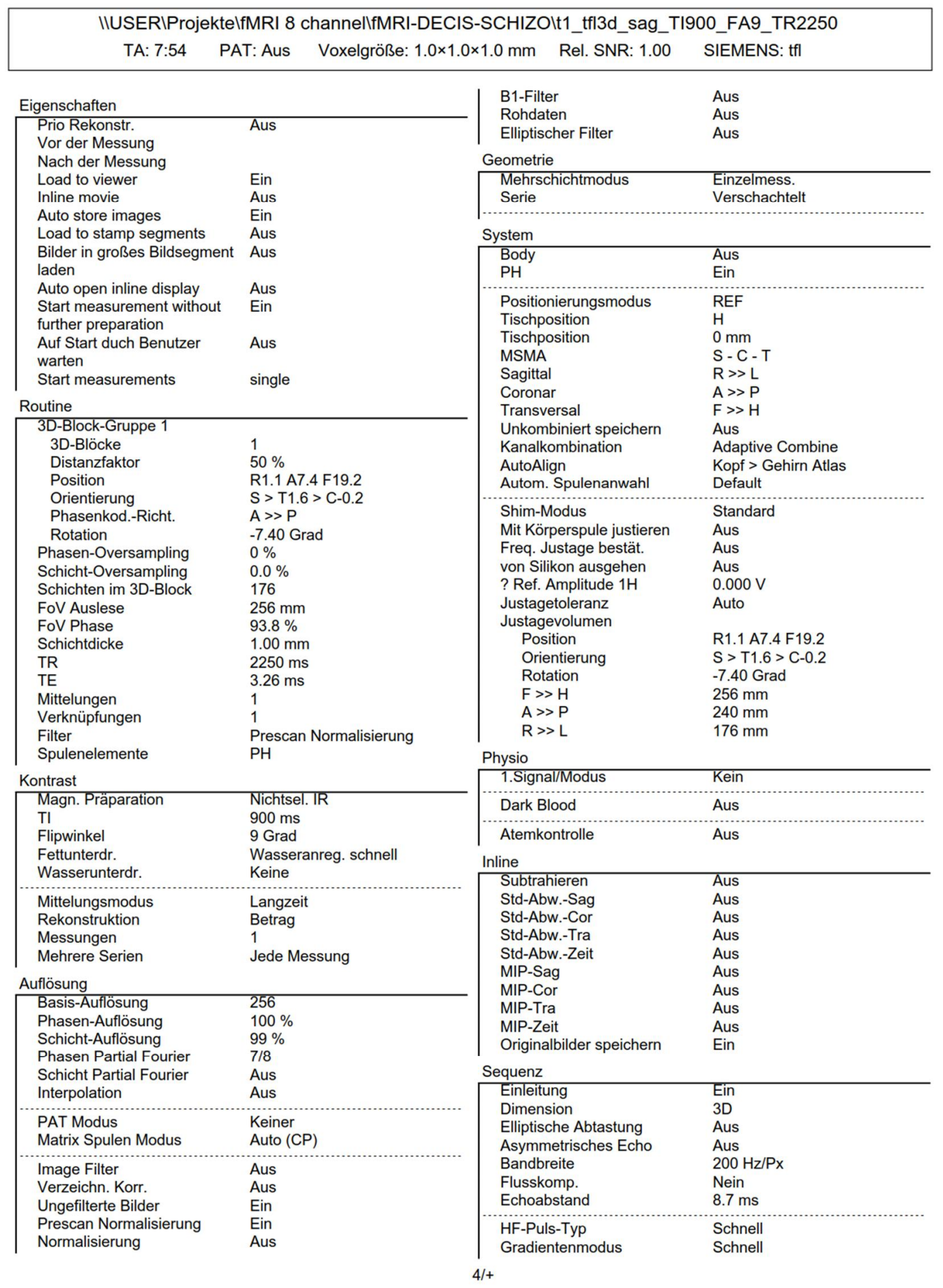


SIEMENS MAGNETOM TrioTim syngo MR B17

Anregung Nichtsel.

HF-Spoiler Ein

5/+ 
SIEMENS MAGNETOM TrioTim syngo MR B17

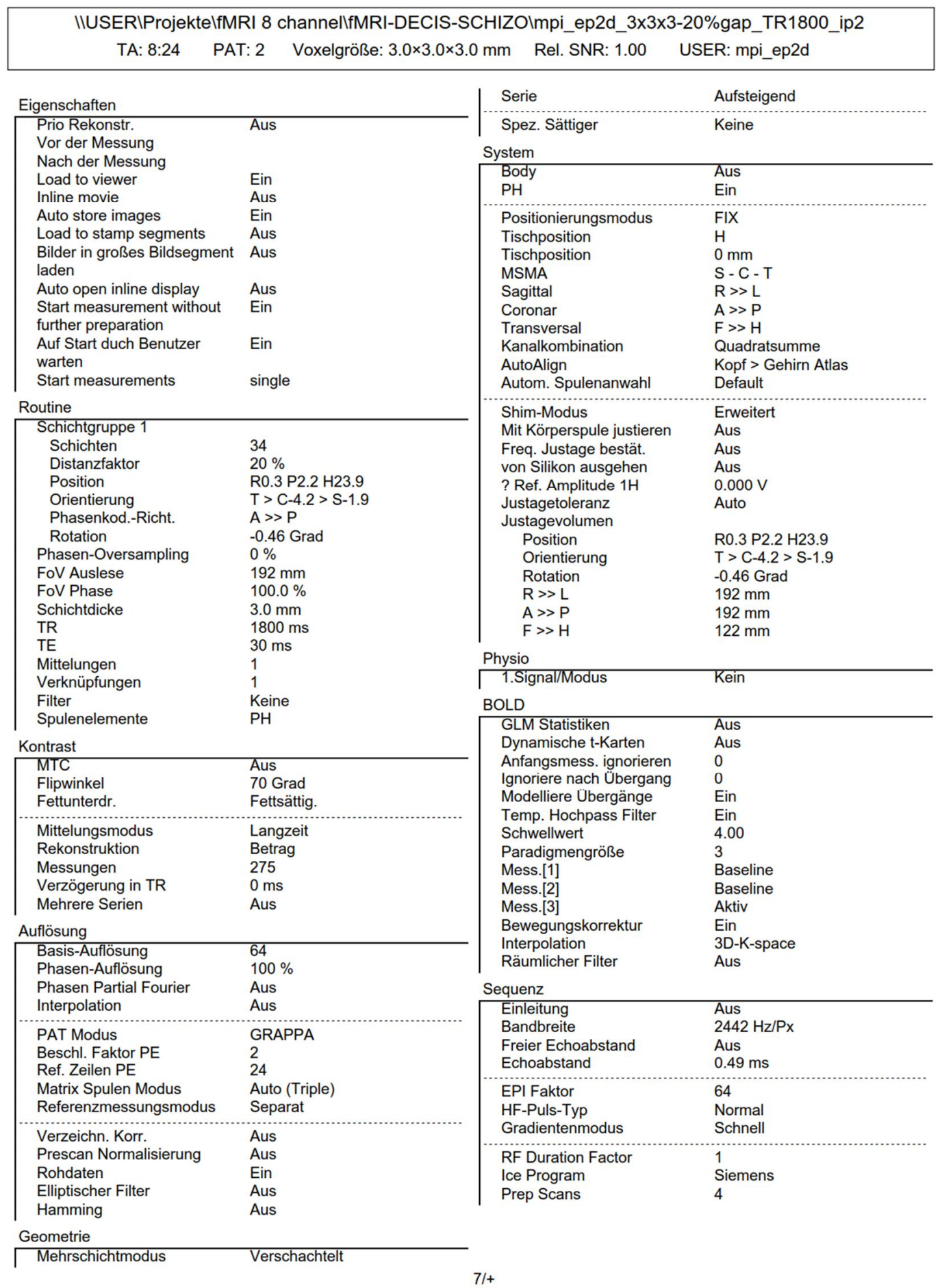




\subsection{Fragebögen}

\subsubsection{Positive and Negative Syndrome Scale (PANSS)}

PID:

Studie:

Untersuchungsdatum:

\section{Positiv und negativ Syndrom Skala (PANSS)}

Positivskala:

$\begin{array}{ll}\text { P1 } & \text { Wahnideen: } \\ \text { P2 } & \text { Formale Denkstörungen: } \\ \text { P3 } & \text { Halluzinationen: } \\ \text { P4 } & \text { Erregung: } \\ \text { P5 } & \text { Größenideen: } \\ \text { P6 } & \text { Mißtrauen/Verfolgungsideen: } \\ \text { P7 } & \text { Feindseligkeit: }\end{array}$

Negativskala:

N1 Affektverflachung:

N2 Emotionaler Rückzug:

N3 Mangelnder affektiver Rapport:

N4 Soziale Passivität und Apathie:

N5 Schwierigkeiten beim abstrakten Denken:

N6 Mangel an Spontaneität und Flüssigkeit der Sprache:

N7 Stereotype Gedanken

Skala der allgemeinen Psychopathologie:

G1 Sorge um die Gesundheit:

G2 Angst:

G3 Schuldgefühle:

G4 Anspannung:

G5 Manierismen und unnatürliche Körperhaltung:

G6 Depression:

G7 Motorische Verlangsamung:

G8 Unkooperatives Verhalten:

G9 Ungewöhnliche Denkinhalte:

G10 Desorientiertheit:

G11 Mangelnde Aufmerksamkeit:

G12 Mangel an Urteilsfähigkeit und Einsicht:

G13 Willensschwäche:

G14 Schwache Impulskontrolle:

G15 Befangenheit:

G16 Aktive soziale Meidung:

ICD 10:

DSM IV: 


\subsubsection{Clinical Global Impression Scale (CGI)}

\section{CGI (Clinical Global Impression Scale)}

PID:

Studie:

Untersuchungsdatum:

(Fragen 2 u. 3 müssen bei Erstbewertungen ausgeklammert werden, indem man 0 (nicht beurteilbar) ankreuzt)

\section{Schweregrad der Krankheit}

Ziehen Sie Ihren gesamten Erfahrungsschatz an dieser Art von Kranken in Betracht, und geben Sie an, wie hoch Sie den jetzigen Grad der seelischen Erkrankung des Patienten einschätzen.

$0 \quad$ Nicht beurteilbar.

Patient ist überhaupt nicht krank.

Patient ist ein Grenzfall psychiatrischer Erkrankung.

Patient ist nur leicht krank.

Patient ist mäßig krank.

Patient ist deutlich krank.

Patient ist schwer krank.

Patient gehört zu den extrem schwer Kranken.

\section{Gesamtbeurteilung der Zustandsänderung}

Beurteilen Sie dabei die Zustandsänderung insgesamt, also nicht nur das Ergebnis der Medikamentenbehandlung. Bitte vergleichen Sie den jetzigen Zustand des Patienten mit dem zu Beginn der Studie, und geben Sie an, inwieweit sich das Krankheitsbild des Patienten geändert hat.

Nicht beurteilbar.

Zustand ist sehr viel besser.

Zustand ist viel besser.

Zustand ist nur wenig besser.

Zustand ist unverändert.

Zustand ist etwas schlechter.

Zustand ist viel schlechter.

Zustand ist sehr viel schlechter. 


\section{Wirksamkeitsindex}

Beurteilen Sie das Verhältnis von therapeutischem Effekt und auftretenden Nebenwirkungen.

\subsection{Therapeutische Wirksamkeit (gewünschte Arzneiwirkungen)}

$0 \quad$ Nicht beurteilbar.

1 Sehr gut - Umfassende Besserung. Vollständige oder fast vollständige Remission aller Symptome.

Mäßig - Deutliche Besserung. Teilweise Remission der Symptome.

Gering - Leichte Besserung. Eine weitere Behandlung des Patienten ist dennoch nötig.

Zustand ist unverändert oder verschlechtert.

3.2. Unerwünschte Wirkungen (Therapierisiken)

$0 \quad$ Nicht beurteilbar.

1 Keine.

2 Beeinträchtigungen des Patienten nicht wesentlich

3 Beeinträchtigungen des Patienten wesentlich.

4 Überwiegen die therapeutische Wirksamkeit. 


\subsubsection{Montgomery-Asberg Depression Scale (MADRS)}

\section{MADRS Montgomery Asberg Depression Scale}

\section{Anleitung}

Die Bewertung sollte nach einem Interview durchgeführt werden, das von mehr allgemeinen Fragen ausgeht und zu detaillierten Symptomem führt, um eine genaue Bewertung des Schweregrades zu erreichen. Der Untersucher muß sich entscheiden, ob seine Beurteilung mit der genauen Beschreibung der Stufen 0, 2, 4, 6 übereinstimmt oder dazwischen liegt $(1,3,5)$. Depressive Patienten, die nicht nach den folgenden 10 Items beurteilt werden können, sind extrem selten!

Wenn vom Patienten selbst keine genauen Antworten zu erhalten sind, sollte sich die Beurteilung auf alle relevanten Anhaltspunkte und Informationen aus anderen Quellen in Übereinstimmung mit der üblichen klinischen Erfahrung stützen.

\section{Sichtbare Traurigkeit}

Dieses Item beinhaltet die sich in Sprache, Mimik und Haltung ausdrückende Mutlosigkeit, Niedergeschlagenheit und Verzweiflung.

0
1
2
3
4
5

Keine Traurigkeit.

Sieht niedergeschlagen aus, ist aber ohne Schwierigkeiten aufzuheitern.

Wirkt die meiste Zeit über traurig und unglücklich.

Sieht die ganze Zeit über traurig und unglücklich aus. Extreme Niedergeschlagenheit.

\section{Berichtete Traurigkeit}

Beinhaltet die vom Patienten berichtete traurige Stimmung, gleichgültig ob sich diese sichtbar ausdrückt oder nicht, einschließlich Entmutigung, Niedergeschlagenheit, dem Gefühl der Hilflosigkeit und Hoffnungslosigkeit.. Bewerten Sie nach Stärke, Dauer und dem Ausmaß der Stimmungsbeeinflussbarkeit durch äußere Ereignisse.

Vorübergehende Traurigkeit je nach den Umständen.

Traurig oder mutlos, jedoch ohne Schwierigkeiten aufzuheitern.

Intensives Gefühl der Traurigkeit und Hoffnungslosigkeit. Die Stimmung ist jedoch immer noch durch äußere Umstände beeinflussbar.

Andauernde oder unveränderliche Traurigkeit, Mutlosigkeit oder Hoffnungslosigkeit. 


\begin{abstract}
3. Innere Spannung
Beinhaltet sowohl ein schwer definierbares Gefühl von Missbehagen als auch Gereiztheit, Unruhe, innere Erregung bis hin zu Angst und Panik. Bewerten Sie nach Stärke, Häufigkeit, Dauer und dem Ausmaß, in dem Beruhigung gesucht wird.

$0 \quad$ Leicht. Nur vorübergehende innere Spannung.

2 Gelegentlich Gefühl von Missbehagen und Gereiztheit.

$4 \quad$ Anhaltendes Gefühl innerer Spannung oder Erregung. Kurzzeitige

Panikanfälle, die der Patient nur mit Mühe beherrscht.

5

6 Nicht beherrschbare Angst oder Erregung. Überwältigende Panik.
\end{abstract}

\title{
4. Schlaflosigkeit
}

Beinhaltet die subjektive Erfahrung verminderter Schlafdauer oder -tiefe, verglichen mit dem vorher normalen Schlafverhalten.

$\begin{array}{ll}0 & \text { Schläft wie gewöhnlich. } \\ 1 & \text { Leichte Schwierigkeiten einzuschlafen. Oberflächlicher, unruhiger Schlaf. } \\ 2 & \text { Geringfügig verkürzte Schlafdauer. } \\ 3 & \\ 4 & \text { Schlaf mindestens zwei Stunden verkürzt oder unterbrochen. } \\ 5 & \text { Weniger als 2-3 Stunden Schlaf. }\end{array}$

\section{Appetitverlust}

Beinhaltet das Gefühl der Abnahme des Appetits, verglichen mit dem vorherigen normalen Zustand. Bewerten Sie nach Stärke des Appetitverlusts oder den zum Essen benötigten Zwang.

$\begin{array}{ll}0 & \text { Normaler oder verstärkter Appetit. } \\ 1 & \\ 2 & \text { Geringfügige Appetitminderung. } \\ 3 & \\ 4 & \text { Kein Appetit. Nahrung wie ohne Geschmack. } \\ 5 & \\ 6 & \text { Nur mit Überredung zum Essen zu bewegen. }\end{array}$




\section{Konzentrationsschwierigkeiten}

Beinhaltet Schwierigkeiten der Konzentration, angefangen vom einfachen Sammeln der eigenen Gedanken bis zum völligen Verlust der Konzentrationsfähigkeit. Bewerten Sie nach Stärke, Häufigkeit und Ausmaß der Unfähigkeit zur Konzentration.

\footnotetext{
$0 \quad$ Keine Konzentrationsschwierigkeiten.

2 Gelegentliche Schwierigkeiten, die eigenen Gedanken zu sammeln.

3

4 Schwierigkeiten, sich zu konzentrieren und einen Gedanken festzuhalten.

5

Nicht in der Lage, ohne Schwierigkeiten zu lesen oder ein Gespräch zu führen.
}

\section{Untätigkeit}

Beinhaltet Schwierigkeiten, ,in Schwung zu kommen“ oder Verlangsamung bei Beginn oder Durchführung der täglichen Arbeiten.



\section{Gefühllosigkeit}

Beinhaltet das subjektive Empfinden des verminderten Interesses für die Umgebung oder Aktivitäten, die vorher Freude bereiteten.

Normales Interesse für Umgebung oder für andere Menschen.

Vermindertes Interesse für Aktivitäten, die vorher Freude bereiteten.

Verlust des Interesses für die Umgebung, Verlust der Gefühle für Freunde und Angehörige.

Die Erfahrung der Gefühllosigkeit. Unfähig, Ärger, Freude oder Trauer zu empfinden. Vollständiger oder schmerzhaft empfundener Verlust des Gefühls für nahe Verwandte und Freunde. 


\section{Pessimistische Gedanken}

Beinhaltet Schuldgefühle, Minderwertigkeitsgefühle, Selbstvorwürfe, Versündigungsideen, Reuegefühle und Verarmungsideen.

$\begin{array}{ll}0 & \text { Keine pessimistischen Gedanken. } \\ 1 & \begin{array}{l}\text { Zeitweise Gedanken, ,versagt zu haben“, Selbstvorwürfe und } \\ 2\end{array} \\ 3 & \text { Selbsterniedrigungen. } \\ 4 & \begin{array}{l}\text { Beständige Selbstanklagen. Eindeutige, aber logisch noch haltbare Schuld- und } \\ \text { Versündigungsideen. Zunehmend pessimistisch in Bezug auf die Zukunft. }\end{array} \\ 5 & \begin{array}{l}\text { Verarmungswahn, Reuegefühl nicht wiedergutzumachender Sünden und } \\ 6\end{array} \\ & \text { Schuld. Selbstanklagen, die logisch absurd, jedoch unkorrigierbar sind. }\end{array}$

\section{Selbstmordgedanken}

Beinhaltet das Gefühl, das Leben sei nicht mehr lebenswert, der natürliche Tod sei eine Erlösung, Selbstmordgedanken und Vorbereitung zum Selbstmord.Selbstmordversuche sollten die Bewertung nicht direkt beeinflussen. Freude am Leben oder die Ansicht, dass man im Leben die Dinge nehmen muß, wie sie kommen.

1

Lebensmüde. Nur zeitweise Selbstmordgedanken.

Lieber tot. Selbstmordgedanken sind häufig. Selbstmord wird als möglicher Ausweg angesehen, jedoch keine genauen Pläne oder Absichten.

Deutliche Selbstmordpläne und -absichten, falls sich eine Gelegenheit bietet. Aktive Vorbereitung zum Selbstmord.

Gesamtwert:

PID:

Studie:

Untersuchungsdatum: 


\section{Literaturverzeichnis}

Abi-Dargham A, Gil R, Krystal J, Baldwin RM, Seibyl JP, Bowers M, van Dyck CH, Charney DS, Innis RB, Laruelle M (1998): Increased Striatal Dopamine Transmission in Schizophrenia: Confirmation in a Second Cohort. Am J Psychiatry $\underline{155}, 761-767$

Abi-Dargham A, Rodenhiser J, Printz D, Zea-Ponce Y, Gil R, Kegeles LS, Weiss R, Cooper TB, Mann JJ, Heertum RLV, et al. (2000): Increased baseline occupancy of D 2 receptors by dopamine in schizophrenia. Proc Natl Acad Sci 97, 8104- 8109

Abler B, Greenhouse I, Ongur D, Walter H, Heckers S (2007): Abnormal Reward System Activation in Mania. Neuropsychopharmacology $\underline{33}$, 2217-2227

Arce E, Leland D S, Miller DA, Simmons AN, Winternheimer KC, Paulus MP (2006): Individuals with schizophrenia present hypo- and hyperactivation during implicit cueing in an inhibitory task. NeuroImage 32, 704-713

Barch D M, Carter CS, Braver TS, Sabb FW, MacD onald A, Noll D C, Cohen JD (2001): Selective D eficits in Prefrontal Cortex Function in Medication-Naive Patients With Schizophrenia. Arch G en Psychiatry $\underline{58}$, 280-288

Bellgrove MA, Hester R, Garavan H (2004): The functional neuroanatomical correlates of response variability: evidence from a response inhibition task. Neuropsychologia $\underline{42}, 1910-1916$

Bleuler E: D ementia praecox, oder G ruppe der Schizophrenien. D euticke, Leipzig 1911

D erntl B, Habel U, Schneider F (2010): Funktionelle Magnetresonanztomographie in der Psychiatrie und Psychotherapie. Nervenarzt 81, 16-23

Derrfuss J, Brass M, von Cramon YD (2004): Cognitive control in the posterior frontolateral cortex: evidence from common activations in task coordination, interference control, and working memory. NeuroImage 23, 604-612

D iekhof EK, Gruber O (2010): When D esire Collides with Reason: Functional Interactions between Anteroventral Prefrontal Cortex and Nucleus Accumbens Underlie the Human Ability to Resist Impulsive Desires. J Neurosci 30, 1488-1493

D iekhof EK, G eier K, Falkai P, G ruber O (2011): Fear is only as deep as the mind allows: A coordinate-based meta-analysis of neuroimaging studies on the regulation of negative affect. NeuroImage $\underline{58,275-285}$ 
Diekhof EK, Keil M, Obst KU, Henseler I, D echent P, Falkai P, Gruber O (2012a): A functional neuroimaging study assessing gender differences in the neural mechanisms underlying the ability to resist impulsive desires. Brain Res $\underline{1473,63-}$ 77

Diekhof EK, Nerenberg L, Falkai P, D echent P, Baudewig J, G ruber O (2012b): Impulsive personality and the ability to resist immediate reward: An fMRI study examining interindividual differences in the neural mechanisms underlying self-control. Hum Brain Mapp 33, 2768-2784

Diekhof EK, Kaps L, Falkai P, Gruber O (2012c): The role of the human ventral striatum and the medial orbitofrontal cortex in the representation of reward magnitude - An activation likelihood estimation meta-analysis of neuroimaging studies of passive reward expectancy and outcome processing. Neuropsychologia 50, 1252-1266

D owd EC, Barch D M (2012): Pavlovian Reward Prediction and Receipt in Schizophrenia: Relationship to Anhedonia. PLOS ONE 7, e35622

Fanelli A (2013): Funktionell-hirnbildgebende Untersuchung zu endophänotypischen Markern bei erstgradigen Angehörigen schizophrener Patienten. Med. Diss. Göttingen

Friston KJ, Buechel C, Fink GR, Morris J, Rolls E, Dolan RJ (1997): Psychophysiological and Modulatory Interactions in Neuroimaging. NeuroImage 6, 218-229

Grimm O, Heinz A, Walter H, Kirsch P, Erk S, Haddad L, Plichta MM, RomanczukSeiferth N, Pöhland L, Mohnke S, et al. (2014): Striatal Response to Reward Anticipation: Evidence for a Systems-Level Intermediate Phenotype for Schizophrenia. JAMA Psychiatry 71, 531-539

Gruber O (2001): Effects of domain-specific interference on brain activation associated with verbal working memory task performance. Cereb Cortex 11, 1047-1055

Gruber O, von Cramon DY (2001): Domain-specific distribution of working memory processes along human prefrontal and parietal cortices: a functional magnetic resonance imaging study. Neurosci Lett 297, 29-32

Gruber O, von Cramon DY (2003): The functional neuroanatomy of human working memory revisited: Evidence from 3-T fMRI studies using classical domain-specific interference tasks. NeuroImage 19, 797-809 
Gruber O, Goschke T (2004): Executive control emerging from dynamic interactions between brain systems mediating language, working memory and attentional processes. Acta Psychol (Amst) 115, 105-121

Gruber O, Falkai P (2009): Von der Identifizierung neurofunktioneller Systeme zur Individualisierung der Therapie schizophrener Störungen. Nervenarzt 80, 12-21

Gruber O, Melcher T, Diekhof EK, Karch S, Falkai P, Goschke T (2009): Brain mechanisms associated with background monitoring of the environment for potentially significant sensory events. Brain Cogn $\underline{69}$, 559-564

Haber SN, Knutson B (2010): The Reward Circuit: Linking Primate Anatomy and Human Imaging. Neuropsychopharmacology 35, 4-26

Huettel SA, Song AW, McCarthy G: Functional Magnetic Resonance Imaging. 3. Auflage; Sinauer Associates, Sunderland 2014

Juckel G, Schlagenhauf F, Koslowski M, Wüstenberg T, Villringer A, Knutson B, Wrase J, Heinz A (2006a): Dysfunction of ventral striatal reward prediction in schizophrenia. NeuroImage 29 , 409-416

Juckel G, Schlagenhauf F, Koslowski M, Filonov D, Wüstenberg T, Villringer A, Knutson B, Kienast T, G allinat J, Wrase J, Heinz A (2006b): Dysfunction of ventral striatal reward prediction in schizophrenic patients treated with typical, not atypical, neuroleptics. Psychopharmacology (Berl) 187, 222-228

Kapur S (2003): Psychosis as a State of Aberrant Salience: A Framework Linking Biology, Phenomenology, and Pharmacology in Schizophrenia. Am J Psychiatry 160, 13-23

Kendler KS, Karkowski LM, Walsh D (1998): The Structure of Psychosis: Latent Class Analysis of Probands From the Roscommon Family Study. Arch Gen Psychiatry $\underline{55}, 492-499$

Knutson B, Fong GW, Adams CM, Varner JL, Hommer D (2001): D issociation of reward anticipation and outcome with event-related fMRI. Neuroreport 12, 3683-3687

Manoach D S (2003): Prefrontal cortex dysfunction during working memory performance in schizophrenia: reconciling discrepant findings. Schizophr Res 60, 285-298

Manoach D S, Press DZ, Thangaraj V, Searl MM, Goff DC, Halpern E, Saper CB, Warach S (1999): Schizophrenic subjects activate dorsolateral prefrontal cortex during a working memory task, as measured by fMRI. Biol Psychiatry $\underline{45}$, 1128-1137 
Manoach DS, Gollub RL, Benson ES, Searl MM, G off D C, Halpern E, Saper CB, Rauch SL (2000): Schizophrenic subjects show aberrant fMRI activation of dorsolateral prefrontal cortex and basal ganglia during working memory performance. Biol Psychiatry 48, 99-109

Marneros A: Schizoaffektive Psychosen. In: Möller H-J, Laux G, Kopfhammer H-P (Hrsg.): Psychiatrie, Psychosomatik, Psychotherapie, Band 2. 4. Auflage; Springer, Berlin 2011, 328-341

Maurer K, Häfner H (2002): Die Östrogenhypothese der Schizophrenie - Übersicht und aktueller Stand der Forschung. J Für Neurol Neurochir Psychiatr 3, 26-34

Melcher T, Gruber O (2006): Oddball and incongruity effects during Stroop task performance: A comparative fMRI study on selective attention. Brain Res 1121, 136- 149

Meyer-Lindenberg A, Miletich RS, Kohn PD, Esposito G, Carson RE, Quarantelli M, Weinberger DR, Berman KF (2002): Reduced prefrontal activity predicts exaggerated striatal dopaminergic function in schizophrenia. Nat Neurosci 5 , 267271

Möller H-J: Schizoaffektive Psychosen. In: Möller H-J, Laux G, Deister A (Hrsg.): Psychiatrie, Psychosomatik und Psychotherapie. 6. Auflage; Thieme, Stuttgart 2015, 156- 183

Möller H-J, Schaub A, Riedel M: Schizophrene Psychosen. In: Möller H-J, Laux G, Kopfhammer H-P (Hrsg.): Psychiatrie, Psychosomatik, Psychotherapie, Band 2. 4. Auflage; Springer, Berlin 2011, 215-324

Moskowitz A, Heim G (2011): Eugen Bleuler's Dementia Praecox or the Group of Schizophrenias (1911): A Centenary Appreciation and Reconsideration. Schizophr Bull 37, 471-479

O 'Connell LA, Hofmann HA (2011): The Vertebrate mesolimbic reward system and social behavior network: A comparative synthesis. J Comp Neurol $\underline{519}$ 3599-3639

O 'Reilly JX, Woolrich MW, Behrens TEJ, Smith SM, Johansen-Berg H (2012): Tools of the trade: psychophysiological interactions and functional connectivity. Soc Cogn Affect Neurosci 7, 604-609 
Pearlson G, Calhoun VD (2009): Convergent approaches for defining functional imaging endophenotypes in schizophrenia. Front Hum Neurosci 3 , 1-11

Perlstein WM, Carter CS, Noll DC, Cohen JD (2001): Relation of Prefrontal Cortex Dysfunction to Working Memory and Symptoms in Schizophrenia. Am J Psychiatry 158, 1105-1113

Rapoport JL, Addington AM, Frangou S, Psych MRC (2005): The neurodevelopmental model of schizophrenia: update 2005. Mol Psychiatry 10, 434-449

Richter A, Petrovic A, Diekhof EK, Trost S, Wolter S, Gruber O (2015): Hyperresponsivity and impaired prefrontal control of the mesolimbic reward system in schizophrenia. J Psychiatr Res 11 , 8-15

Roiser JP, Howes OD, Chaddock CA, Joyce EM, McGuire P (2013): Neural and Behavioral Correlates of Aberrant Salience in Individuals at Risk for Psychosis. Schizophr Bull 39, 1328-1336

Romaniuk L, Honey GD, King JRL, Whalley HC, McIntosh AM, Levita L, Hughes M, Johnstone EC, Day M, Lawrie SM, Hall J (2010): Midbrain Activation D uring Pavlovian Conditioning and Delusional Symptoms in Schizophrenia. Arch Gen Psychiatry $\underline{67}, 1246-1254$

Scarr E, Gibbons AS, Neo J, Udawela M, D ean B (2013): Cholinergic connectivity: it's implications for psychiatric disorders. Front Cell Neurosci 7, 1-26

Schlagenhauf F, Sterzer P, Schmack K, Ballmaier M, Rapp M, Wrase J, Juckel G, G allinat J, Heinz A (2009): Reward Feedback Alterations in Unmedicated Schizophrenia Patients: Relevance for D elusions. Biol Psychiatry 65, 1032-1039

Sesack SR, Grace AA (2009): Cortico-Basal Ganglia Reward Network: Microcircuitry. Neuropsychopharmacology $\underline{35}, 27-47$

Shenton ME, Dickey CC, Frumin M, McCarley RW (2001): A review of MRI findings in schizophrenia. Schizophr Res $\underline{49}, 1-52$

Stephan KE, Baldeweg T, Friston KJ (2006): Synaptic Plasticity and Dysconnection in Schizophrenia. Biol Psychiatry 59, 929-939

Tandon R, Keshavan MS, Nasrallah HA (2008): Schizophrenia, "Just the Facts" What we know in 2008. 2. Epidemiology and etiology. Schizophr Res 102, 1-18 
Tandon R, Nasrallah HA, Keshavan MS (2009): Schizophrenia, "Just the Facts" 4. Clinical features and conceptualization. Schizophr Res 110, 1-23

Tandon R, Nasrallah HA, Keshavan MS (2010): Schizophrenia, "Just the Facts" 5. Treatment and prevention. Past, present, and future. Schizophr Res 122, 1- 23

Wolter S, Petrovic A, Vieker H, Melcher T, Trost S, Diekhof EK, Richter A, Zilles D, Gruber $O$ (2016): Dysregulation within the Prefronto-Parietal BackgroundMonitoring Network in Schizophrenia. J Behav Brain Sci 06, 364

Y oon JH, Minzenberg MJ, Raouf S, D’Esposito M, Carter CS (2013): Impaired PrefrontalBasal Ganglia Functional Connectivity and Substantia Nigra Hyperactivity in Schizophrenia. Biol Psychiatry $\underline{74}, 122-129$ 


\section{Danksagung}

Ich bedanke mich herzlich bei Herm Prof. Dr. Oliver Gruber, Universitätsprofessor für Experimentelle Psychopathologie und Bildgebung am Zentrum für Psychosoziale Medizin der Universität Heidelberg, für die Möglichkeit, die vorliegende Arbeit in seiner damaligen Arbeitsgruppe in Göttingen durchführen zu können. Nicht nur durch die Bereitstellung der erforderlichen Mittel, sondem auch die umfassende Betreuung und die Einführung in das wissenschaftliche Arbeiten waren mir eine große Hilfe.

Mein besonderer Dank geht an Frau Jun.-Prof. Dr. Esther Diekhof für die konkrete Einführung in das verwendetet Paradigma sowie die Auswertung der erhobenen Daten. Ebenso danke ich Frau Maria Keil für ihre Hilfe bei der Organisation und Koordination der sehr zeitaufwendigen Untersuchungen. Weiter bedanke ich mich bei der gesamten damaligen Arbeitsgruppe Systemische Neurowissenschaften der Klinik für Psychiatrie und Psychotherapie der Universitätsmedizin Göttingen. Mein D ank gilt auch den Mitarbeitern der Wissenschaftlichen Serviceeinrichtung MR-Forschung in der Neurologie und Psychiatrie, insbesondere Frau Ilona Pfahlert und Frau Britta Perl, für die tatkräftige Unterstützung bei der D urchführung der MR-Untersuchungen.

Insbesondere geht auch großer Dank an meine Probandinnen und Probanden, ohne welche die vorliegende Arbeit nie zu Stande gekommen wäre. 